\title{
CONDITIONING OF INTERICTAL BEHAVIOURS, BUT NOT ICTAL BEHAVIOURS, SEIZURES, OR AFTERDISCHARGE THRESHOLD, BY KINDLING OF THE AMYGDALA IN RATS
}

\author{
A Thesis Submitted to the College of \\ Graduate Studies and Research \\ In Partial Fulfillment of the Requirements \\ For the Degree of Master of Arts \\ In the Department of Psychology \\ University of Saskatchewan \\ Saskatoon
}

By

JASON P. WAGNER

(c) Copyright Jason P. Wagner, January, 2007. All rights reserved. 


\section{Permission to Use}

In presenting this thesis in partial fulfilment of the requirements for a Postgraduate degree from the University of Saskatchewan, I agree that the Libraries of this University may make it freely available for inspection. I further agree that permission for copying of this thesis in any manner, in whole or in part, for scholarly purposes may be granted by the professor or professors who supervised my thesis work or, in their absence, by the Head of the Department or the Dean of the College in which my thesis work was done. It is understood that any copying or publication or use of this thesis or parts thereof for financial gain shall not be allowed without my written permission. It is also understood that due recognition shall be given to me and to the University of Saskatchewan in any scholarly use which may be made of any material in my thesis.

Requests for permission to copy or to make other use of material in this thesis in whole or part should be addressed to:

Head of the Department of Psychology University of Saskatchewan Saskatoon, Saskatchewan S7N 5A5 


\begin{abstract}
Repeated focal electrical stimulation of the brain results in kindling, the development of generalized seizures that progress in length and severity as more seizures are elicited. Barnes et al. (2001) paired one context (CS+) with kindling stimulation of the amygdala, and another context (CS-) with sham stimulation. They found conditioned anticipatory fear responses in the CS+, a conditioned place aversion to the CS+, and more intense convulsions in the CS+ than in the CS- in a probe trial. The present experiment was an attempt to replicate the findings, and to extend them by recording electroencephalographs (EEG). As well, I tested for conditioned effects on afterdischarge threshold (ADT). Rats received 45 pairings in each context before a conditioned place preference/aversion test, to determine whether the stimulation and seizures were rewarding or punishing. After more pairings, rats received suprathreshold stimulation in each context (switch test). Ictal measures in this test included afterdischarge duration, clonus duration, latency to clonus, class of convulsion, and falls. After more pairings, ADT was measured in each context. I partially replicated the findings of Barnes et al., in that conditioned anticipatory responses and conditioned place aversion were found. However, there were no conditioned effects on any ictal measures, including ADT. I conclude that conditioning is unlikely to play a major role in epileptogenesis.
\end{abstract}




\section{Acknowledgements}

First I would like to thank my supervisor, Michael E. Corcoran. He has contributed significantly to this thesis. His help has ranged from helping me start as a kindling researcher, to providing motivation and valuable guidance, and to intelligent critiques of earlier versions of this thesis. Perhaps most importantly, he has routinely helped me see the bigger picture, and how science really works.

I also value the input of my committee members, Deb Saucier and Lisa Kalynchuk, and wish to thank them. Their input has often provided me with fresh thinking about my project and data.

My lab-mates have been very supportive and helpful, and so I thank them. Ken Wolfe was instrumentally helpful in getting me started in the lab, and remained generous in is help thereafter. Ann Lam, Bonita Ma, Naomi Whelan, Jolly De Guzman, and Joanne Sitarski, have all been helpful, encouraging, and generally good company in my time in the lab. 


\section{Dedication}

To my wife, Susan.

She has been profoundly supportive of me and my work.

I am amazed daily by her love and dedication. 


\section{TABLE OF CONTENTS}

page

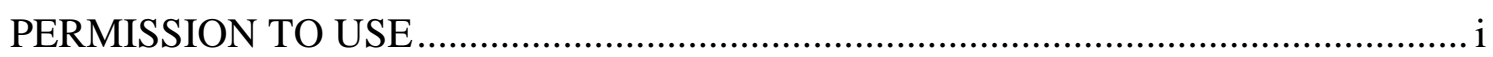

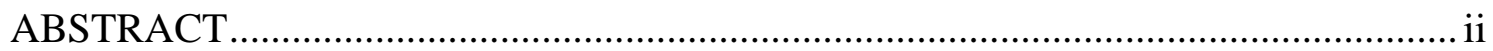

ACKNOWLEDGEMENTS ...............................................................................

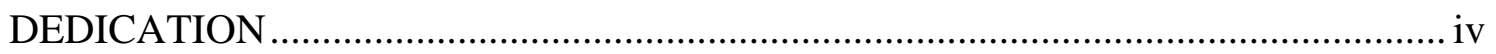

LIST OF FIGURES ................................................................................. vii

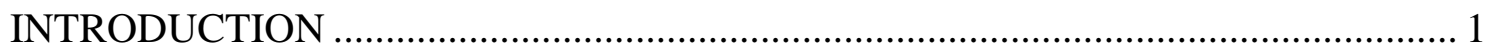

Behavioural Treatments of the Epilepsies …...................................................... 1

Evidence from Experimental Preparations for a Role of Conditioning....................... 3

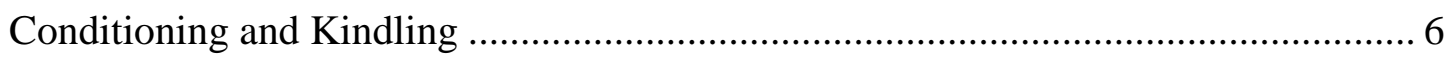

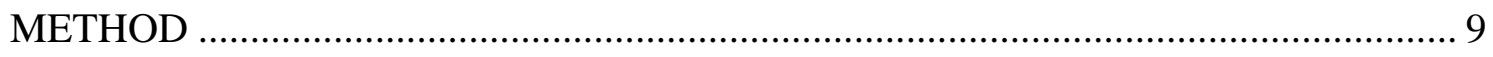

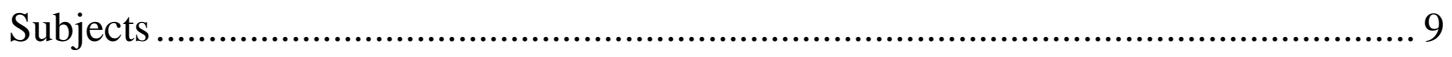

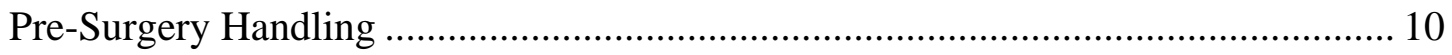

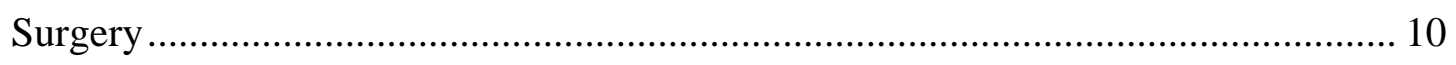

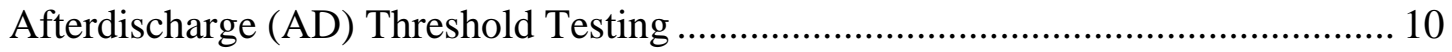

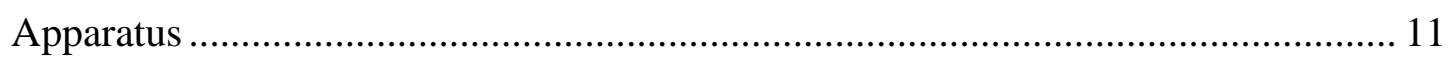

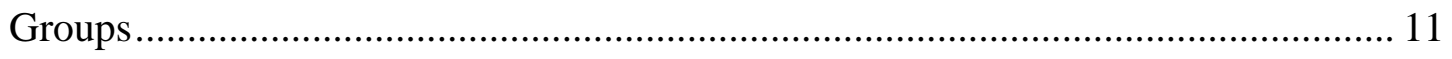

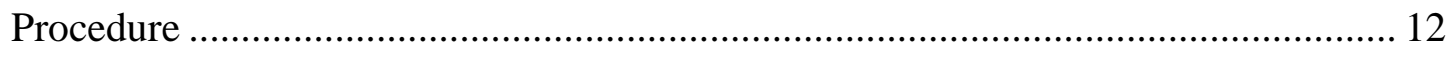

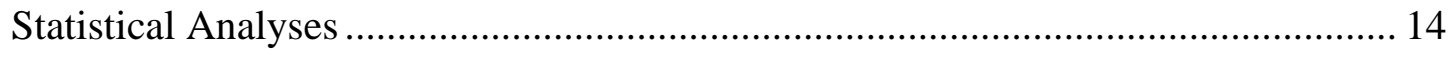

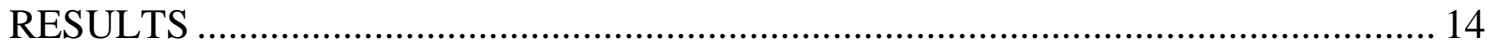

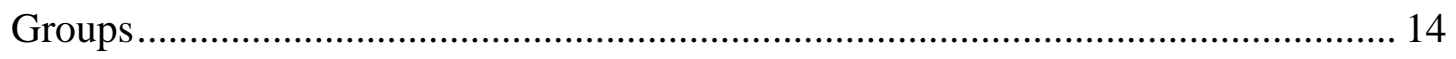

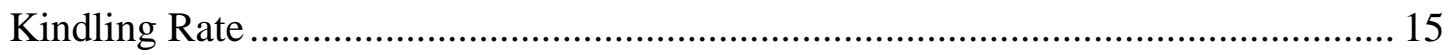




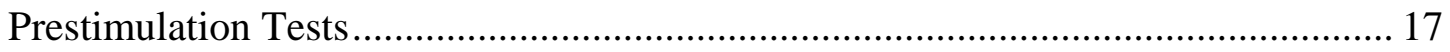

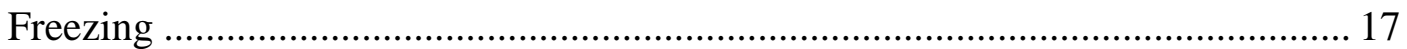

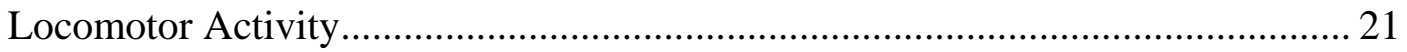

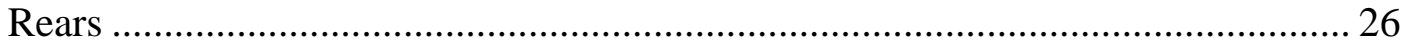

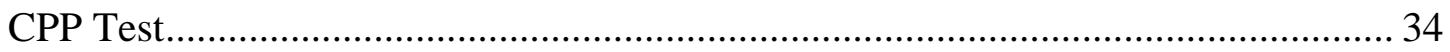

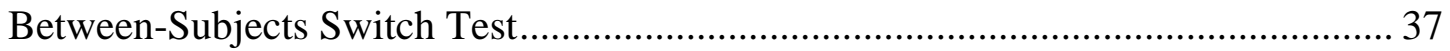

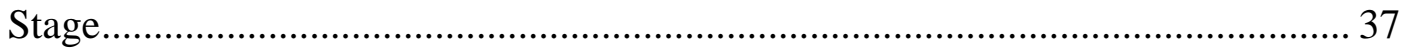

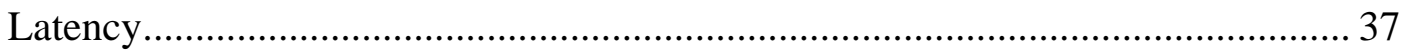

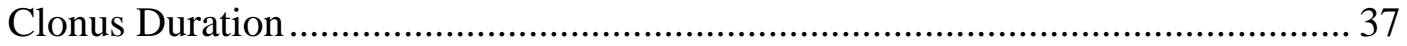

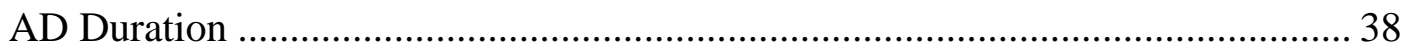

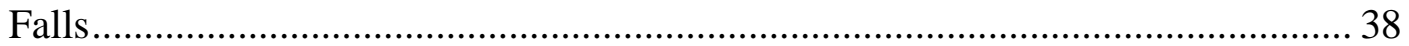

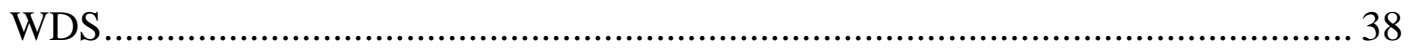

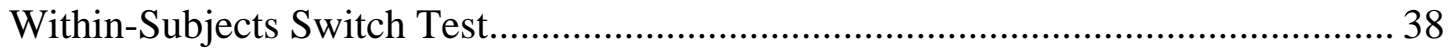

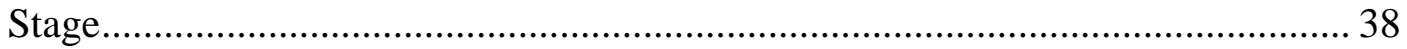

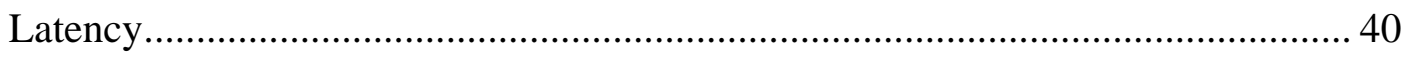

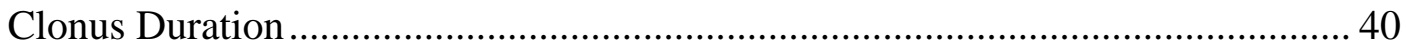

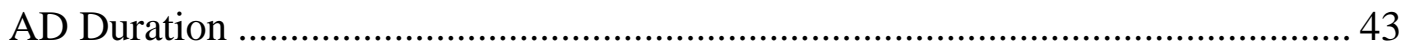

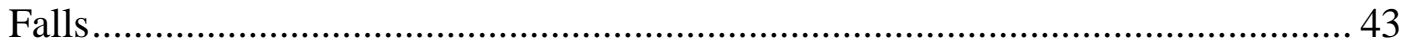

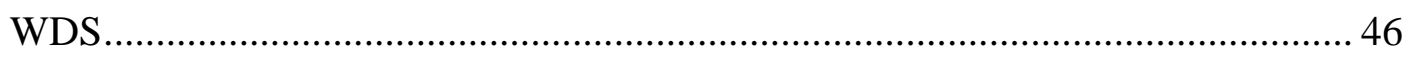

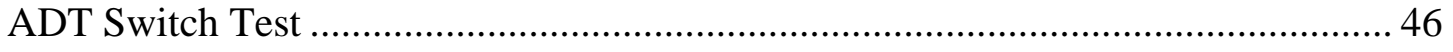

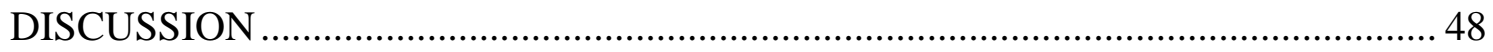

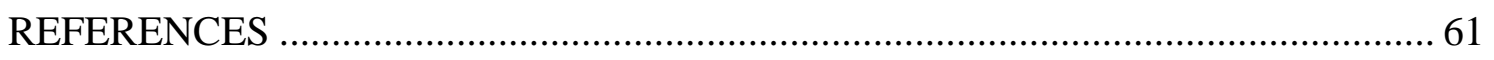

APPENDIX 1: PERMISSION TO REPRINT COPYRIGHTED MATERIAL ............. 70 


\section{LIST OF FIGURES}

Figure

page

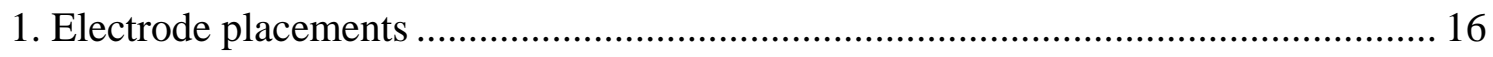

2. Freezing in 16 prestimulation test trials.......................................................... 19

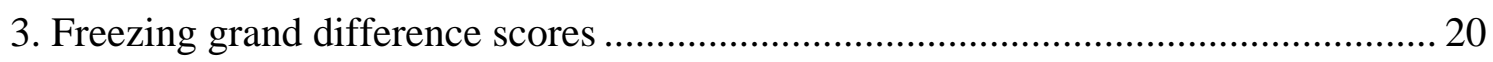

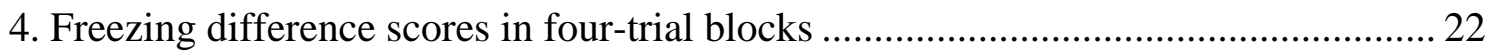

5. Locomotor activity in 16 prestimulation test trials ............................................ 24

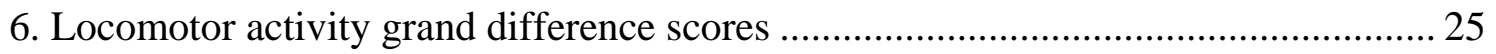

7. Locomotor activity difference scores in four-trial blocks.................................... 27

8. Rearing by White rats in 16 prestimulation test trials......................................... 30

9. Rearing difference scores by White rats in four-trial blocks ................................... 31

10. Rearing by Black rats in 16 prestimulation test trials ......................................... 33

11. Rearing difference scores by Black rats in four-trial blocks.................................. 35

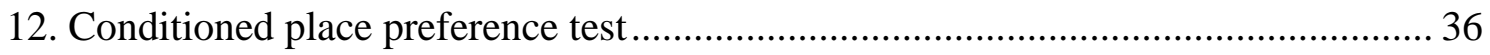

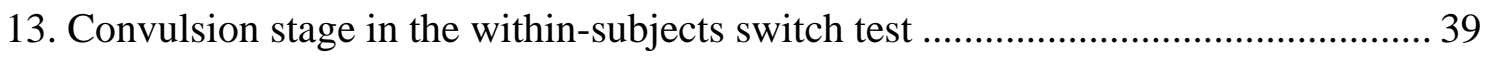

14. Latency to stage 3 convulsion in the within-subjects switch test .......................... 41

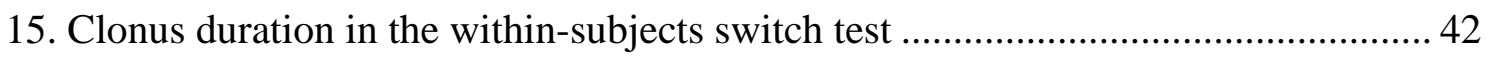

16. Afterdischarge duration in the within-subjects switch test .................................. 44

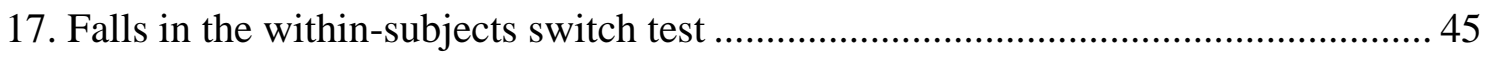

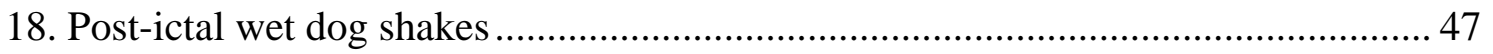

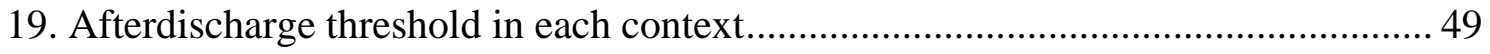

20. Afterdischarge threshold by electrode placement ............................................ 50 
Conditioning of interictal behaviours, but not ictal behaviours, seizures, or afterdischarge threshold, by kindling of the amygdala in rats

Epilepsy is a heterogeneous neurological disorder, which consists of many related syndromes. It has been estimated to affect 50,000,000 people worldwide (World Health Organization, 2001). The common characteristic among types of epilepsy is the spontaneous recurrence of seizures, which are paroxysmal electrical events within the brain. Seizures may arise from diffuse or specific foci within the brain. In cases of specific foci, convulsive symptoms, antecedent states, and interictal semiology may be related to the seizure focus in each individual (Hannesson \& Corcoran, 2000; Helmstaedter et al., 2004), although semiological profiles of different structures often overlap (Helmstaedter et al., 2004; Kalynchuk et al., 1998a; So, 2006).

Antiepileptic drugs (AEDs), also known as antiseizure drugs, are the most common treatment for epilepsy. In most cases, AEDs are effective at controlling seizures. However, in approximately 20-30\% of cases, AEDs are not effective; as well, AEDs commonly have unpleasant, and even debilitating adverse effects (for review, see Nadkarni et al., 2005).

Behavioural Treatments of the Epilepsies

There have been attempts to develop non-pharmacological treatment for epilepsy. These include habituation and extinction techniques for sensory-induced seizures, EEG biosensory feedback, and the application of behaviour-modification techniques to seizure-antecedent behaviours. They have not been well accepted by the neurological community; despite decades of research and keen support from their proponents (e.g., Andrews \& Schonfeld, 1992; Cataldo et al., 1979; Sterman \& Egner, 2006; Wyler et al., 
1976), such treatments rarely receive mention in authoritative descriptions of available epilepsy treatments (Lowenstein, 2005; Nadkarni et al., 2005)

Forster (1969, 1972, 1967, 1964, 1965, 1964a, 1964b) successfully reduced the frequency of sensory-induced seizures through conditioning. Other researchers have had difficulty replicating his findings (Wolf, 2002). Most other early studies of behavioural treatments for epilepsies had serious methodological problems (Krafft \& Poling, 1982).

In some cases, seizures are reliably preceded by certain behaviours (Ng, 2002; Zlutnick et al., 1975) or thoughts (Ritaccio et al., 2002). Some individuals intentionally perform behaviours that elicit seizures (for review, see Ng, 2002). In cases of specific preseizure behaviours, Zlutnick et al. (1975) reported reductions in seizures using behaviour modification techniques targeting the preseizure behaviours. They argued that those behaviours and the seizures formed behavioural chains that could be interrupted (Skinner, 1934).

Another nonpharmaceutic approach to epilepsy treatment is EEG biofeedback, which involves the provision of reinforcement for certain patterns of electrical activity as measured on EEG (Blanchard \& Young, 1974; Egner \& Sterman, 2006; Sterman, 2000; Sterman \& Egner, 2006). Although its proponents argue that EEG biofeedback is a viable treatment for epilepsy (Egner \& Sterman, 2006; Sterman, 2000; Sterman \& Egner, 2006), biofeedback is controversial (Cott et al., 1979; Quy et al., 1979), and has not been fully accepted by the medical community.

Insight into the potential role of the environment and conditioning (learning) in epileptogenesis has come from studies of experimental epilepsy in animals. I will review 
this evidence, with particular emphasis on kindling, the preparation I used in my own research.

\section{Evidence from Experimental Preparations for a Role of Conditioning}

Repeated focal electrical stimulation of some discrete sites in the brain results in kindling, the development of generalized seizures that progress in length and severity as more seizures are elicited. Kindling was first reported and described by Goddard (Goddard, 1967) and Racine (Racine, 1972a, 1972b). It is a good animal preparation of partial complex epilepsy, in that the seizures originate from a discrete focus, and eventually generalize to other parts of the brain. Additionally, seizures kindled from some sites are accompanied by a loss of equilibrium, as evidenced by falling, and this is considered to be analogous to an alteration of consciousness in a human complex seizure.

Kindling is also thought to be related to learning and memory. Whereas both kindling and learning are thought to involve neuroplastic changes, those induced by kindling are considered pathological, and not representative of normal learning and memory (Saucier \& Corcoran, 2003).

Many neuroanatomical structures have been kindled, but those in the limbic system have been studied the most. This includes the amygdala, which was the structure of interest in the first reports of kindling (Goddard, 1967; Racine, 1972a, 1972b).

Although originally used by Burdach to describe what we now call the Basolateral Amygdala (BLA), the term amygdala is often used to describe that and several other interconnected nearby nuclei (Sah et al., 2003). The amygdala receives input from a wide variety of cortical and subcortical sources, including input from all sensory modalities. 
Likewise, it projects to a wide variety of cortical and subcortical sites (for a thorough review, see Sah et al., 2003).

The amygdala has long been recognized for its involvement in emotion. Initially, this recognition came from studies showing deficits in fear and anxiety after experimental lesions (e.g., Goldstein, 1965; e.g., Kluver \& Bucy, 1939; Robinson, 1963). Stimulation of the amygdala has been shown to result in increases in fear-related behaviour (e.g., Hunsperger, 1963; Maclean \& Delgado, 1953). The amygdala is also involved in general arousal, appetite, and sexual behaviour (Goddard, 1964).

For fear-related behaviours, there may be some functional differentiation between the central and basolateral nuclei of the amygdala. Using experimental lesions, the basolateral nucleus has been shown to mediate conditioned avoidance behaviour, whereas the central nucleus has been shown to mediate fear-induced suppression of behaviour (Killcross et al., 1997).

When limbic sites are kindled, the convulsions reliably progress through characteristic stages, each associated with characteristic motor movements. Racine (1972b) first described five stages (Stage 1: facial clonus; Stage 2: facial clonus with head-nodding; Stage 3: unilateral forelimb clonus; Stage 4: rearing and bilateral forelimb clonus; Stage 5: as with Stage 4, with one fall). This scale was later extended by Pinel and Rovner (1978), to include three additional stages that occur with relatively extended kindling of the amygdala (Stage 6: as with Stage 5, with multiple falls; Stage 7: as with Stage 6, with running or jumping fits; Stage 8: a convulsion with one or more periods of tonus). 
It is generally believed that the kindled state is stable, that is, that animals kindled to generalized seizures will predictably emit such seizures when later stimulated (Goddard et al., 1969). As well, it has been believed that each animal's ADT is indicative of internal physiological states close to the electrode tip (Racine, 1972a), and not of learned associations.

Kindling of some structures results in behavioural changes specific to those structures (Barnes et al., 2005; Barnes et al., 2001; Barnes et al., 2003; Hannesson \& Corcoran, 2000; Kalynchuk et al., 1998a; D. C. McIntyre, 1979; D. C. McIntyre \& Molino, 1972; Wig et al., 2002). For example, kindling of the hippocampus results in deficits in spatial cognition (Gilbert et al., 2000; Gilbert et al., 1996; Hannesson \& Corcoran, 2000; Hannesson et al., 2004; Leung et al., 1996; Lopes da Silva et al., 1986), but not in other forms of cognition not thought to be mediated by the hippocampus (Hannesson et al., 2001).

Kindling of the amygdala usually results in changes in anxiety-related behaviour (R. Adamec \& Shallow, 2000; R. E. Adamec \& Morgan, 1994; Kalynchuk, 2000; Kalynchuk et al., 1998a, 1999; Kalynchuk et al., 1998b; Kalynchuk et al., 1997; D. C. McIntyre, 1970, 1979; Wintink et al., 2003). Kindling of the amygdala also disrupts emotional learning (D. C. McIntyre, 1970, 1979). When rats are kindled extensively, with up to 100 amygdaloid stimulations, they are more defensive in a resident-intruder test, they are more resistant to capture by an unfamiliar experimenter, they display heightened anxiety-like behaviour in the open field, and they display unusual anxiety like behaviour in the elevated-plus maze, where they sometimes jump off the maze presumably to escape it (Kalynchuk et al., 1998a, 1999; Kalynchuk et al., 1998b; Kalynchuk et al., 
1997; Wintink et al., 2003). These effects are reliably produced by extended amygdaloid kindling, but they can also be produced by extended hippocampal kindling (Kalynchuk et al., 1998a), suggesting that extensive kindling may decrease the focal specificity of behavioural effects. Thus after many generalized seizures, behaviours mediated by multiple related structures may be affected.

\section{Conditioning and Kindling}

In recent years, Steven J. Barnes and colleagues (Barnes et al., 2005; Barnes et al., 2004; Barnes et al., 2001; Barnes et al., 2006; Barnes et al., 2003; Wig et al., 2002) have used kindling, or some aspect thereof, as the unconditioned stimulus in a classical conditioning paradigm. They have identified conditioned effects on interictal behaviours, as well as conditioned effects on ictal measures. Their original paradigm (Barnes et al., 2001) used a place conditioning procedure, in which rats were kindled by amygdaloid stimulation in one context (CS+), and received sham stimulation in another (CS-). This involved many (53 in total) pairings of each type. In the CS+, rats developed conditioned anticipatory defensive responses, including decreased locomotion, decreased rearing, and increased freezing, as compared to their baseline of behaviour in the CS-. As well, rats showed a conditioned place aversion to the CS+ when given a choice between the two contexts.

The place aversion described by Barnes et al. (2001) contrasts with the results of Corcoran, Lanius, and Duren (1992), who used fewer pairings and did not find a significant conditioned place preference or aversion. It also contrasts with the results of Paredes, Muzzi, Aguirre, and Romero (2000), who found a conditioned place preference for the context in which rats were placed after generalized kindled seizures. Paredes et al. 
argued that the rewarding effects indicated by the place preference occur during the postictal period. If the results of both Barnes et al. and Paredes et al. are correct, it would seem that the aversive aspects of amygdaloid kindling or amygdaloid-kindled seizures are more salient than the rewarding aspects of the postictal state produced thereby.

Given the role of the amygdala in fear, anxiety, and emotional memory, Barnes et al.'s (2001) results may not seem surprising. However, they do contradict the long-held view that generalized seizures have amnestic effects. For example, amygdaloid-kindled generalized convulsions have been shown to produce amnestic effects on conditioned emotional response (D. C. McIntyre, 1970).

The provocative results from Barnes et al.'s (2001) original paper and later papers (Barnes et al., 2004; Barnes et al., 2001; Barnes et al., 2006; Barnes et al., 2003) are those showing conditioned effects on ictal measures, such as convulsion stage and duration. The researchers found that, with amydaloid-kindled convulsions, the convulsions were less severe when elicited in the CS- than in the CS+. This was shown using "switch tests" (Barnes et al., 2001), p1067), which came after many pairings of the CS+ with kindled convulsions, and the CS- with sham stimulation. Barnes's results are in contrast to those of Freeman and Mikulka (1986), who failed to find a contextually-conditioned effect on AD duration with amygdaloid kindling. In any case, Barnes's results could be interpreted to indicate that the environment and conditioning play a key role in kindling and the intensity of kindled convulsions (Barnes et al., 2001).

The potentially important role of the environment and conditioning as a general mechanism in kindling is challenged by Barnes's finding that when other forebrain structures are kindled, different results are found (Barnes et al., 2005; Barnes et al., 
2003). When seizures were elicited by anterior neocortical kindling, there was no evidence of conditioned defensiveness or place preference or aversion, and convulsions were less severe in the CS+ than in the CS- (Barnes et al., 2003). Barnes et al. (2005) also kindled three different sites in the hippocampal formation in a similar experiment. Perirhinal kindling produced conditioned anticipatory freezing and decreased locomotion. Unfortunately, rearing data were notably absent from that experiment. Ventral hippocampal kindling only produced conditioned freezing, but not a conditioned decrease in locomotion, when the experiment was extended to include many more pairings. Dorsal hippocampal kindling did not produce any conditioned anticipatory defensiveness, even with the extended experimental design. A conditioned place aversion was found with perirhinal and ventral hippocampal kindling, but not with dorsal hippocampal kindling.

Barnes et al.'s (2005) reasonable explanation for the differential effects on anticipatory defensive behaviours is that, because ventral and dorsal hippocampal kindling require a large number of stimulations before generalized convulsions develop, it is likely the generalized convulsions that serve as the relevant unconditioned stimulus that is, that conditioning does not occur until generalized convulsions have been kindled. Alternatively, the hippocampus may not be sufficiently involved in anxiety and fear relative to the amygdala to produce conditioned defensiveness. The perirhinal cortex is also arguably anatomically and functionally dissociable from the hippocampal formation (see Paxinos, 1995). Perhaps more important, there were no conditioned effects on ictal measures. Ostensibly, this means that hippocampal seizures are not as easily conditioned. However, it is possible that conditioning of ictal measures is not as reliable as some other 
experiments have suggested (Barnes et al., 2004; Barnes et al., 2001; Barnes et al., 2006; Barnes et al., 2003).

In the present experiment I attempted to replicate Barnes et al. (2001), and to extend their findings by recording electroencephalographs (EEG), thereby permitting testing for conditioned effects on afterdischarge (AD) and afterdischarge threshold (ADT). Because triggering of $\mathrm{AD}$ is fundamental to amygdaloid kindling and kindled convulsions, recording EEG seemed to be particularly relevant in this experiment. Note that Barnes did not record EEG in his experiments. As well, I hypothesized that the conditioned effects on ictal measures found by Barnes and colleagues may have been related to conditioned differences in ADT, and I therefore included an ADT switch test in the present experiment. I hypothesised that I would replicate the findings of Barnes et al. (2001), and that I would find conditioned effects on AD and ADT.

\section{Method}

\section{Subjects}

Subjects were 41 male Long-Evans rats from Charles Rivers Laboratories (St. Constant, Quebec, Canada), weighing 370g to 540g at the time of surgery. They were housed in groups of two to four prior to surgery, and housed individually thereafter. Food and water were freely available. Lights followed a 12h:12h light-dark schedule, with lights on at 7am; experimental procedures were conducted during the light portion of the cycle. Housing and experimental procedures were in accordance with the guidelines of the Canadian Council on Animal Care. 


\section{Pre-surgery Handling}

Starting 5 to 7 days after arrival, rats were handled for 1.5 to 3 minutes each, once per day, for five days. Handling consisted of gentle stroking, picking up, holding, and talking to each rat.

Surgery

Rats were anesthetized with isoflurane (IsoFlo) administered by inhalation (5\% initial, 2.5\% thereafter) and placed in a stereotaxic apparatus. During surgery, Anafen ${ }^{\circledR}$ was injected s.c. $(5 \mathrm{mg} / \mathrm{kg})$ for post-operative analgesia. Two bipolar electrodes, made from $127 \mu \mathrm{m}$-diameter enamel-insulated nichrome wire, were implanted at the following coordinates: $2.6 \mathrm{~mm}$ posterior, $4.5 \mathrm{~mm}$ lateral, and $9.1 \mathrm{~mm}$ ventral from bregma, with the skull level. Coordinates were derived from Paxinos and Watson (1998). Four stainless steel dental screws, to which a ground electrode was mounted, were fixed to the skull surface. The electrode assembly was affixed to the skull using dental acrylic. Hibitaine ( $)$ was applied to the area of the incision to minimize infection. Starting 1 to 2 weeks after surgery, rats were handled as before for five days. Afterdischarge (AD) Threshold Testing

Prior to experimental pairings, AD threshold (ADT) was determined for each rat. The rat was placed in a clear acrylic chamber that was different from the apparatus used for the rest of the experiment. Electrical stimulation was generated on a Grass S8800 stimulator and consisted of a 1-s train of balanced biphasic square-wave pulses $1.0 \mathrm{~ms}$ in duration and delivered at $60 \mathrm{pp} / \mathrm{s}$ to one of the two electrodes, starting at $20 \mu \mathrm{A}$ and increasing by $10 \mu \mathrm{A}$ every minute, to a maximum of $100 \mu \mathrm{A}$. If $\mathrm{AD}$ lasting at least $5 \mathrm{sec}$ was not elicited, the schedule was repeated using the other electrode. If no AD was 
elicited, the rat was returned to its home cage. The next day, the rat was returned to the same chamber, and stimulation was applied in the same manner as before, but using the following intensity schedule: $120 \mu \mathrm{A}, 140 \mu \mathrm{A}, 160 \mu \mathrm{A}, 180 \mu \mathrm{A}, 200 \mu \mathrm{A}, 220 \mu \mathrm{A}$, $250 \mu \mathrm{A}, 300 \mu \mathrm{A}, 350 \mu \mathrm{A}, 400 \mu \mathrm{A}$. The first electrode in each animal to elicit an AD using this protocol was subsequently used as the stimulating electrode for kindling. This was intended to select an electrode that was likely to be in the BLA, which tends to have lower ADTs than adjacent nuclei (Mohapel et al., 1996).

\section{Apparatus}

The conditioned place preference apparatus was of a rectangular box shape, with two distinct halves (contexts) separable by removable dividers. The apparatus measured $48 \mathrm{~cm}(\mathrm{w})$ by $79 \mathrm{~cm}(\mathrm{l})$ by $25 \mathrm{~cm}(\mathrm{~h})$ overall. With dividers in place, each context measured $48 \mathrm{~cm}(\mathrm{w})$ by $37 \mathrm{~cm}(\mathrm{l})$ by $25 \mathrm{~cm}(\mathrm{~h})$. One context was constructed of white acrylic panels, with recycled-paper bedding (CareFresh ${ }^{\circledR}$ ) on the floor. The other context was constructed of black acrylic panels, and the floor was left bare. The entire apparatus had two clear acrylic lids, mounted with hinges. When closed, the lids left a $3 \mathrm{~cm}$ gap running the length of the apparatus, perpendicular to the dividers, which allowed the stimulation and recording lead to reach the rat, without restricting the rat's movements.

Groups

Rats were randomly assigned to either the WHITE or BLACK group, corresponding to the context that served as their CS+. WHITE rats received CS+ trials in the white half of the apparatus and CS- trials in the black half. BLACK rats received 
$\mathrm{CS}+$ trials in the black half of the apparatus and CS- trials in the White half of the apparatus.

Procedure

Rats received a total of 63 pairings of each of two types. In CS+ trials, each rat was placed in its CS+. After 30sec in the context, the rat received a train of kindling stimulation. In CS- trials, the rat was placed in its CS-, but received no stimulation. Each trial lasted 180s, and there were two trials daily. The order of these trials was pseudorandom, with the criterion that each rat received no more than three trials of one type in a row. On every fourth day, each rat received one trial of each type, in counterbalanced order. The purpose of these days was to examine the rats' behaviour in each context in the prestimulation interval, during the first 30sec after being placed in the apparatus. The behaviours examined included the number of rears, the total duration of freezing episodes longer than five sec, and locomotor activity as measured by gridline crosses. Behavioural convulsions were scored using Pinel and Rovner’s (1978) eightpoint scale (Stage 1: facial clonus; Stage 2: facial clonus with head-nodding; Stage 3: unilateral forelimb clonus; Stage 4: rearing and bilateral forelimb clonus; Stage 5: as with Stage 4, with one fall; Stage 6: as with Stage 5, with multiple falls; Stage 7: as with Stage 6, with running or jumping fits; Stage 8: a convulsion one or more periods of tonus). As well, latency to clonus, clonus duration, the number of falls, and AD duration were recorded for each seizure.

After 45 pairings, rats were placed in the apparatus, with dividers removed. They were thus able to move freely between the two contexts. This assessed their preference 
between the two contexts, to test whether amygdaloid kindling is associated with affective consequences, either punishing or rewarding.

After eight more pairings of each type, rats received a switch test (Barnes et al., 2001). Half of the rats received stimulation in their CS+ context, and the other half received stimulation in their CS- context. Comparison between the two groups constituted the between-groups switch test. Assignment to the groups was counterbalanced, in that it was not predicted by assignment to context (WHITE or BLACK) or by the counterbalancing schedule used for prestimulation test days. Rats that received a stimulation in the CS+ in that test received a stimulation in their CSapproximately 4 to 5 hrs later. Comparison between each rat's CS+ trial and CS- trial on that day constituted the within-subjects switch test.

After eight more pairings of each type, the rats' ADT was determined in each context. Stimulation intensity was gradually increased until an $\mathrm{AD}$ of at least 5 sec was elicited, using the following schedule: $20 \mu \mathrm{A}, 30 \mu \mathrm{A}, 40 \mu \mathrm{A}, 50 \mu \mathrm{A}, 60 \mu \mathrm{A}, 70 \mu \mathrm{A}, 80 \mu \mathrm{A}$, $90 \mu \mathrm{A}, 100 \mu \mathrm{A}, 120 \mu \mathrm{A}, 140 \mu \mathrm{A}, 160 \mu \mathrm{A}, 180 \mu \mathrm{A}, 200 \mu \mathrm{A}, 220 \mu \mathrm{A}, 250 \mu \mathrm{A}, 300 \mu \mathrm{A}, 350 \mu \mathrm{A}$, $400 \mu \mathrm{A}$. This procedure was completed in one session for each context, exclusively employing the same electrode used for kindling. Half of the rats were first tested in their $\mathrm{CS}+$, and the other half were first tested in their CS-. After approximately $24 \mathrm{hr}$, the rats were tested in the other context. Assignment to this order was counterbalanced such that it was not predicted by assignment to context (WHITE or BLACK), the counterbalancing schedule used for prestimulation test days, or assignment to the switch test conditions.

Rats were euthanized with Euthanyl ${ }^{\circledR}$, and were then perfused transcardially with phosphate-buffered saline (PBS) and 4\% paraformaldehyde in PBS (PFA). Brains were 
stored in PFA for 1 to 3 days. Each brain was then stored in 30\% sucrose in PBS, until it sunk (2 to 4 days). The brains were sectioned at $40 \mu \mathrm{m}$ using a freezing microtome. The sections were either stored in PBS at $4^{\circ} \mathrm{C}$ for less than $24 \mathrm{hr}$ or stored in cryoprotectant solution at $-20^{\circ} \mathrm{C}$ for up to 5 days. Every second section was then mounted on gelatincoated slides, and sections were stained with cresyl violet. Electrode placements were verified using a light microscope and a reference atlas (Paxinos \& Watson, 1998). Statistical Analyses

Data were analyzed using univariate or mixed-model Analyses of Variance (ANOVAs), where appropriate. Within groups of tests (e.g., Prestimulation Tests, or Within-Subjects Switch Test), a Bonferroni correction was used. This was done to correct for the increased probability of Type I error associated with multiple tests. The correction divides the desired $\alpha$ level by the number of tests (e.g., three, for the Prestimulation Tests), to obtain the $\alpha$ level to be used for the individual tests. I did not use a Multivariate Analysis of Variance (MANOVA), because I was interested in effects on individual dependent variables, rather than relations among the dependent variables. This approach is supported by Huberty and Morris (1989). Finally, significant interactions from individual ANOVAs were followed by $t$-tests. These tests were also adjusted using a Bonferroni correction; the $\alpha$ level was adjusted for the number of $t$-tests that followed each interaction.

\section{Results}

\section{Groups}

Of the 41 rats, only the data from the 28 rats with placements in the BLA $(n=20)$ or central nucleus of the amygdala $(n=8)$ were used in this experiment (Figure 1 
describes the placements). One rat died during surgery, and another died in recovery. One rat was found, post-mortem, to have a trans-cranial infection causing a mass effect, and its data were excluded from analyses. Another rat was found to have a cystic cavity, approximately $2 \mathrm{~mm}^{3}$ at the tip of the kindling electrode, and was therefore excluded. As well, 5 rats dislodged their electrode pedestals during the course of the experiment. There were 4 rats with kindling-electrode tips located elsewhere in the amygdala. That group was excluded from analyses, because its electrode placements were heterogeneously distributed throughout the extended amygdala. Of the included rats, 17 had the white context as their CS+ (White group), and 11 had the black context as their CS+ (Black group).

\section{Kindling Rate}

Two measures were used to quantify each rat's rate of kindling: the number of kindling stimulations required to elicit the first stage 5 or greater seizure, and the number of stimulations required to elicit five consecutive stage 5 seizures. Four rats did not have five consecutive stage 5 seizures during the entire experiment, and were assigned values of either 62 or 63 , equal to the total number of stimulations they received. Each of these dependent variables was analyzed using $2(\mathrm{CS}+) * 2$ (Placement) Univariate ANOVAs, with a Bonferroni correction requiring a $p$ value of $\alpha / 2=0.05 / 2=0.025$. The $p$ values are reported uncorrected. The omnibus ANOVA analyzing the number of stimulations to the first stage 5 did not reach significance; no main effects or interactions occurred, $F(3,24)$ $=1.052, p=0.388$. The omnibus ANOVA analyzing the number of stimulations required for five consecutive stage 5 seizures also did not reach significance; there were no main 
Figure 1. Electrode placements from BLA rats (stars) and Central Amygdala rats (circles). For rats in which both electrodes were used for stimulation $(\mathrm{n}=3)$, both placements are shown. Images were adapted from Paxinos and Watson (1997, used with permission). Measurements are relative to Bregma.
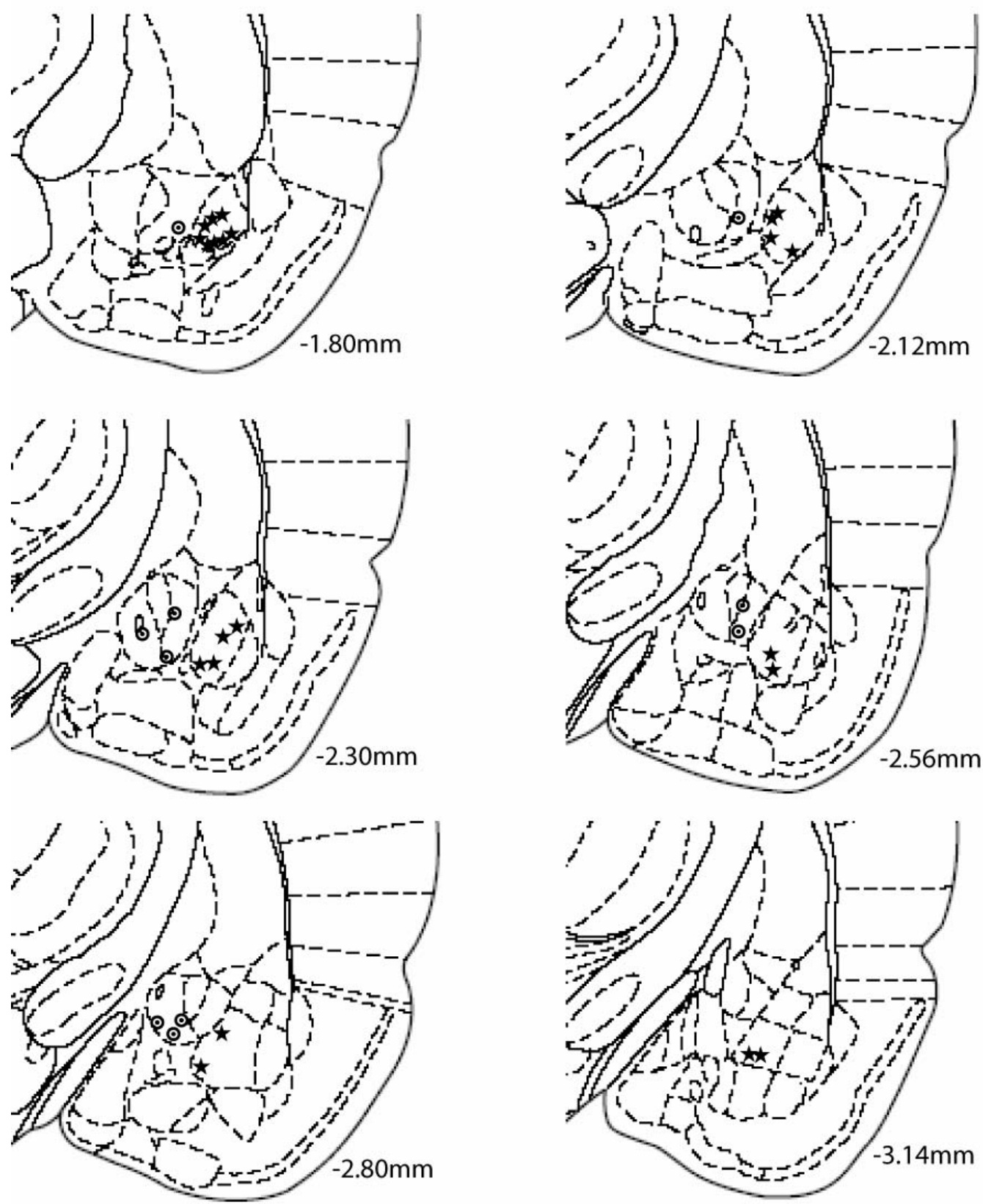
effects or interactions, $F<1$. Thus kindling was unaffected by the environment (White or Black) in which stimulation was applied.

Prestimulation Tests

Freezing, Locomotor Activity, and Rears data from prestimulation test trials were entered into separate 16 (Trial) $* 2$ (Context) $* 2$ (Placement) $* 2(\mathrm{CS}+)$ mixed-model ANOVAs. A Bonferroni correction was used to correct for an increased probability of Type I error; this required a $p$ value of $\alpha / 3=0.05 / 3=0.0167$. All $p$ values are reported uncorrected.

Freezing. Mauchly's Test of Sphericity revealed that the assumption of sphericity was violated for Trial, $W(119)<0.0005, p<0.0005$. As well, the assumption of sphericity was found to be violated for the Trial * Context interaction term, $W(119)<$ 0.0005, $p<0.0005$. The Huynh-Feldt $\varepsilon$ correction was therefore used for all tests involving the Trial term $(\varepsilon=0.567)$, and for all tests involving the Trial * Context term ( $\varepsilon$ $=0.469)$.

The main effect of Trial approached statistical significance, indicating there was likely at least one difference that approached significance among the 16 trials, $F(8.498$, 203.960) $=2.147, p=0.030$.

There was a significant main effect of Context, with rats freezing more in the CS+ (52.26 +/- $9.69 \mathrm{~s})$ than in the CS- $(8.91+/-2.74 \mathrm{~s})$ during the prestimulation test trials, $F(1,24)=17.165, p<0.0005$. The main effect of Placement was not statistically significant, and thus BLA rats (63.02 +/- 15.19 s) did not differ from Central Amygdala rats (56.54 +/- $15.29 \mathrm{~s})$ in time spent freezing during the prestimulation test trials, $F<1$. The main effect of CS+ was also not statistically significant, and thus White rats (74.26 
+/- $15.03 \mathrm{~s})$ did not differ significantly from Black rats (40.92 +/- $17.08 \mathrm{~s})$ in time spent freezing, $F(1,24)=1.641, p=0.212$.

A variety of two-way interactions were statistically insignificant: Trial * Placement, $F<1$; Trial * CS+, $F<1$; , Context * Placement, $F<1$; and Placement $* C S+$, $F<1$. The Context $*$ CS + interaction approached significance, $F(1,24)=4.017, p=$ 0.056. As illustrated in Figure 2, the Trial * Context interaction was significant, $F(7.029$, 1.8.687) $=2.787, p=0.009$.

A variety of three-way interactions were also statistically insignificant: Trial * Placement * CS+, F<1; Context * Placement * CS+, $F<1$; Trial * Context * Placement, $F<1$, Trial $*$ Context $*$ CS + interaction, $F<1$; Trial * Context * Placement * $\mathrm{CS}+, F<1$.

Although the Context * CS+ interaction did not reach significance, further analysis was conducted to examine a possible differential effect of CS+ assignment to contextually conditioned differences in freezing. Each rat's total duration of freezing in each context during prestimulation test trials was computed. These totals were then used to compute a grand difference score, representing the total time spent freezing in the CS+ minus compared to that in the CS-. A positive score would therefore indicate a greater total duration of freezing in the CS+ than in the CS-. An independent-samples t-test was then computed to compare White rats to Black rats on the grand difference score (see Figure 3). This $t$-test revealed that White rats showed a greater difference in freezing between their CS+ and CS- $(57.70+/-11.10 \mathrm{~s})$ than Black rats $(21.17+/-9.55 \mathrm{~s}), t(26)=$ 2.307, $p=0.029$ (two-tailed). To follow up the Trial * Context interaction, the 16 prestimulation test trials were grouped into four blocks of four consecutive trials each. A 
Figure 2. Freezing in each context during the 16 prestimulation test trials. All rats were tested in each context, in counterbalanced order. Data are averaged across both levels of Placement.

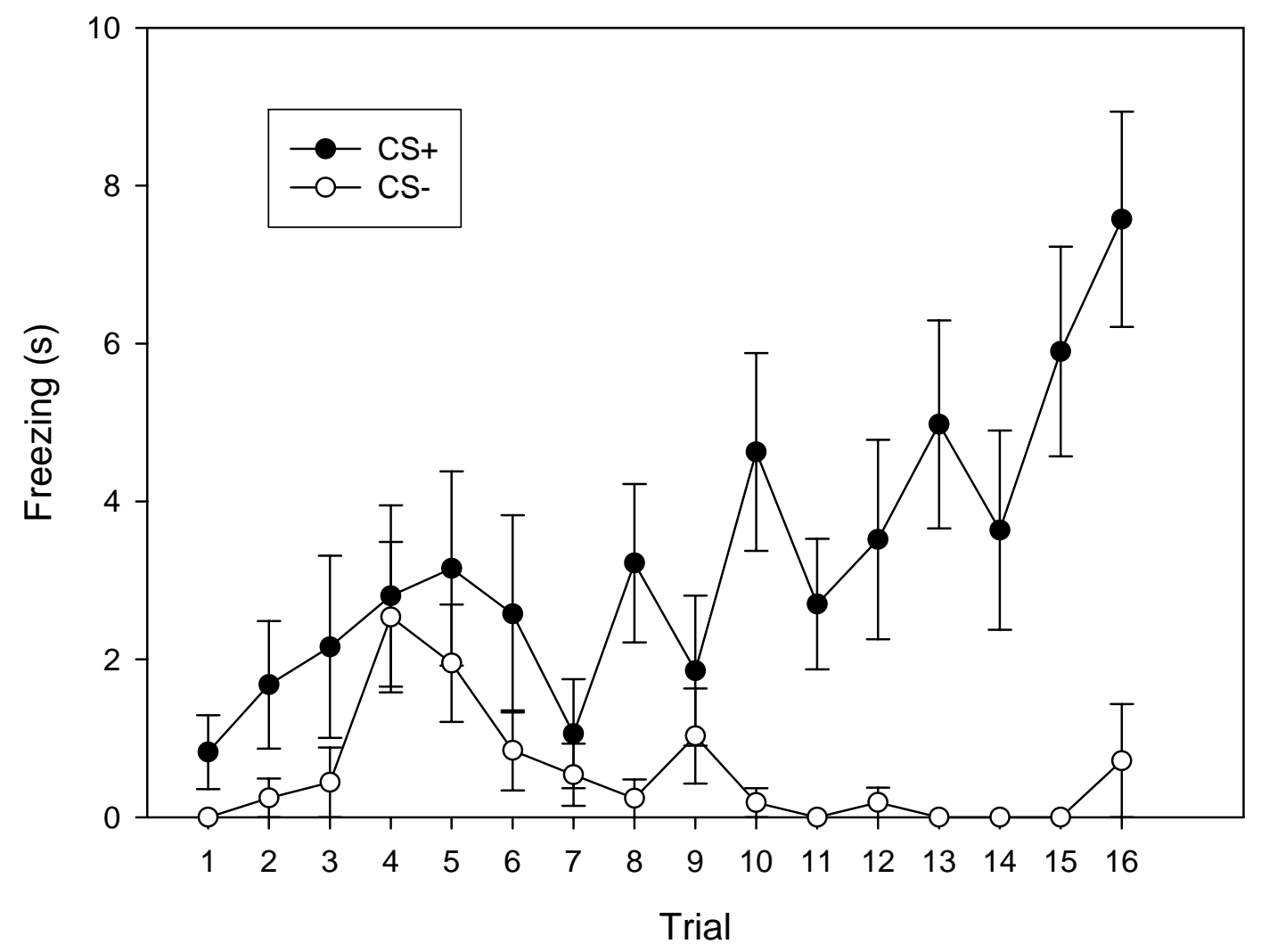


Figure 3. Freezing grand difference scores, based on all prestimulation test trials, for rats in each of the CS+ assignment groups. The difference scores were calculated by subtracting the duration of all freezing in the CS- from the duration of all freezing in the CS+. Data are averaged across both levels of Placement. White rats showed a significantly larger difference in freezing between their CS+ and CS- than did Black rats, $p=0.029$.

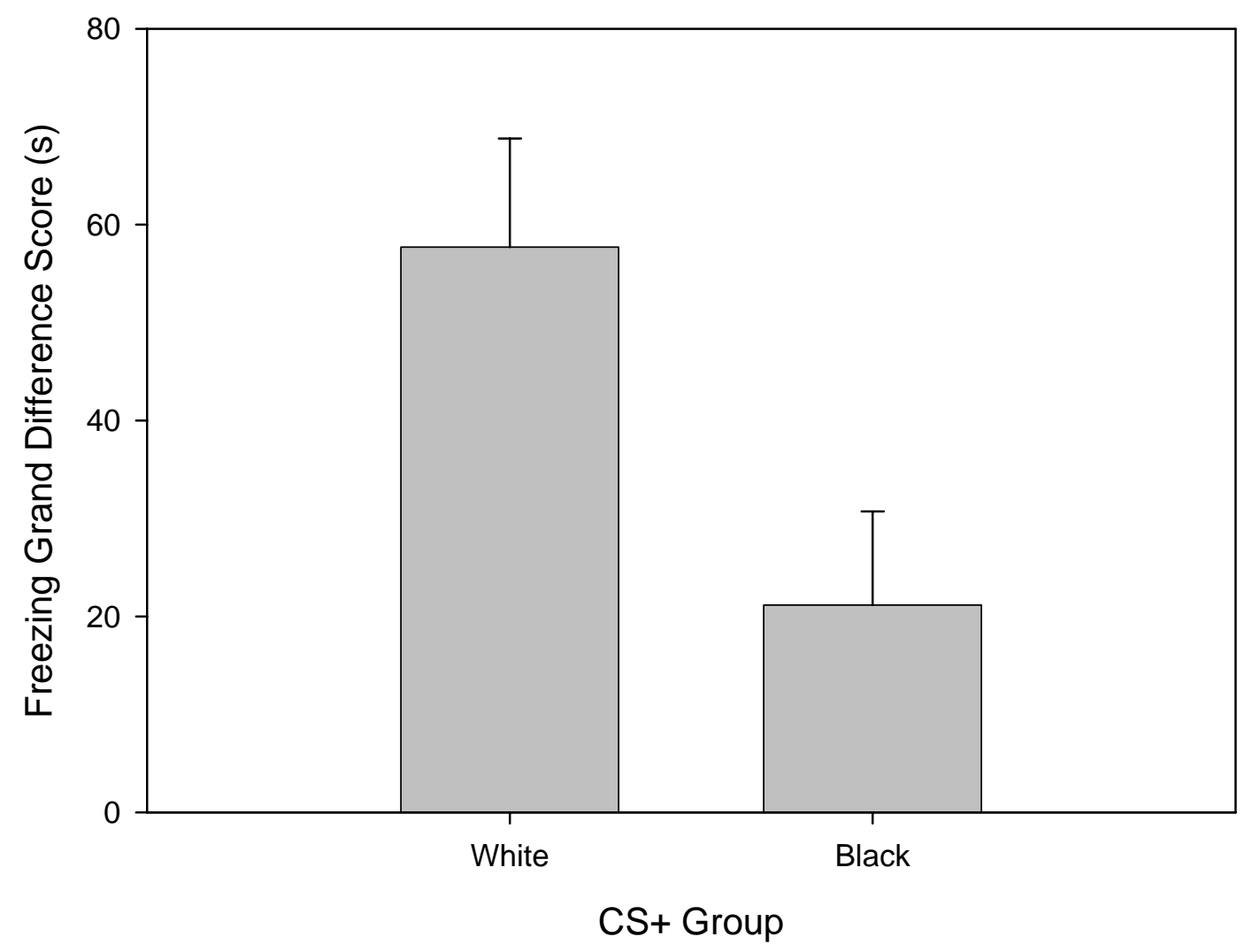


sum of time spent freezing in each context was then computed for each block. A difference score was then computed for each block, using the formula of time spent freezing in the CS+ minus time spent freezing in the CS-; a positive score therefore indicated more time spent freezing in the CS+ than in the CS- for that block. These difference scores are shown in Figure 4. Three planned repeated-measures $t$-tests were computed, comparing the difference score from the first block to the difference scores from each of the other three blocks. A significant difference wherein the latter block's difference score was higher than the first block's difference score therefore indicates a differential increase in freezing in the CS+ relative to the CS-. A Bonferroni correction requiring $\alpha / 3=0.05 / 3=0.0167$ was used. All $p$ values are reported uncorrected, and are one-tailed.

The first (4.24 +/- $2.02 \mathrm{~s})$ and second (6.43+/- 3.09 s) blocks were not significantly different, with rats showing similar differential degrees of freezing in the two contexts for the first two blocks, $t(27)=-1.019, p=0.158$. The difference between the first block and the third block (11.30 +/- 3.61 s) approached statistical significance; rats showed a nonsignificantly greater degree of freezing in the CS+ relative to the CS- in the third block than in the first block, $t(27)=-1.929, p=0.032$. The fourth block (21.37 +/- $4.06 \mathrm{~s}$ ) was, however, significantly different from the first block, with rats showing a greater degree of freezing the CS+ relative to the CS- in the fourth block than in the first block, $t(27)=-3.529, p=0.001$.

Locomotor activity. There was significant main effect of trial, indicating that there was at least one significant difference in locomotor activity among the 16 trials, $F(15$, $360)=6.535, p<0.0005$. There was also a significant main effect of Context, with rats 
Figure 4. Freezing difference scores from each of the four blocks of prestimulation test trials. Data are averaged across both levels of Placement and CS+. Asterisk denotes a significant difference when compared to the difference score from Block 1.

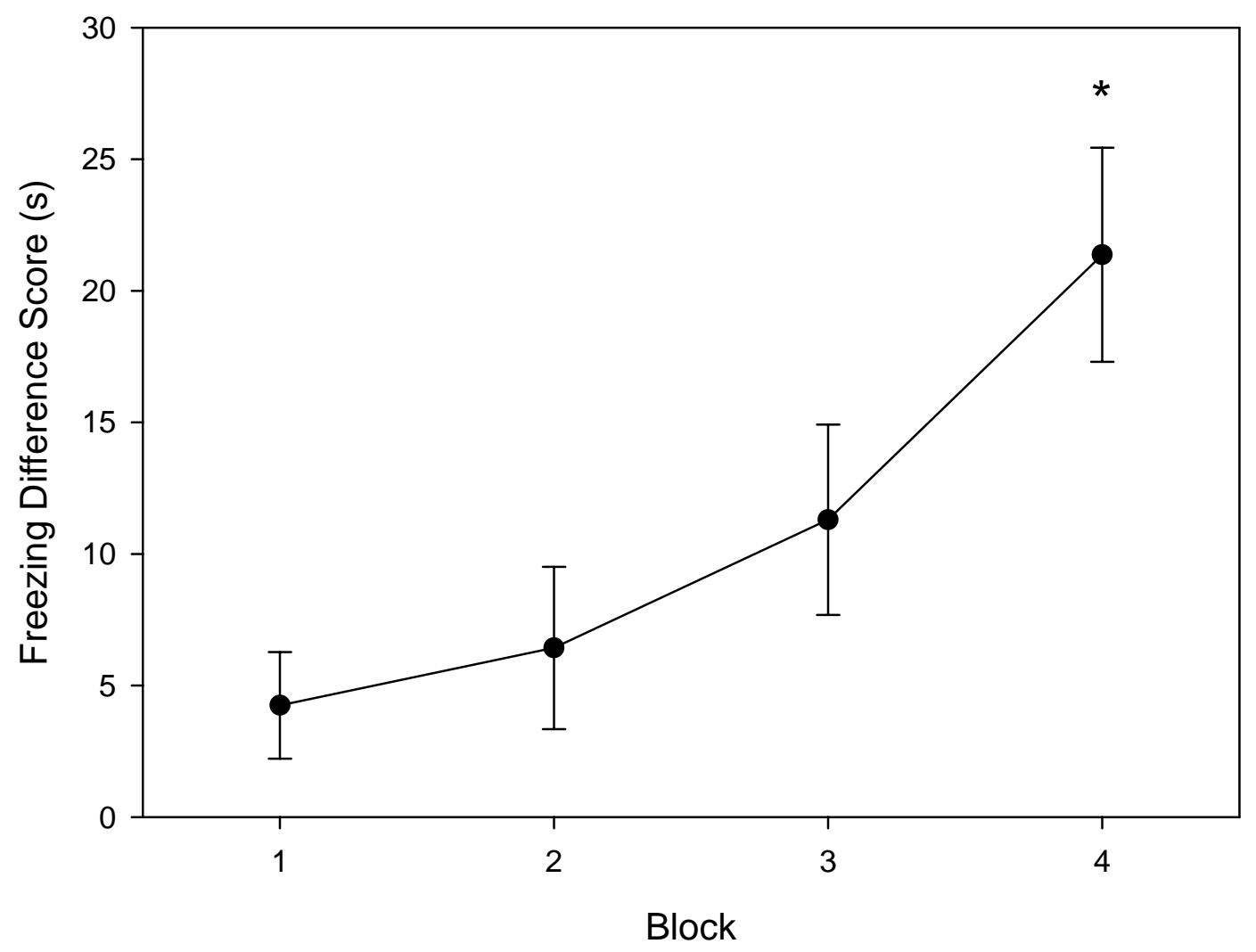


making fewer line crosses in the CS+ $(36.38+/-4.48)$ than in the CS- $(103.54+/-7.92)$, $F(1,24)=69.076, p<0.0005$. There was no significant main effect of CS+, indicating that White rats $(138.40+/-11.39)$ did not differ from Black rats $(142.27+/$ - 20.96) in the number of lines crossed, $F<1$. There was also no significant main effect of Placement, indicating that BLA rats (147.54 +/- 10.39) did not cross more or fewer lines than Central Amygdala rats (120.88+/- 26.27), $F<1$.

The Trial * CS+ interaction was not significant, $F(15,360)=1.093, p=0.361$. There was no significant Trial * Placement interaction, $F<1$. The Context $*$ CS + interaction approached significance, $F(1,24)=5.710, p=0.025$. There was no significant Context * Placement interaction, $F<1$. There was a significant Trial * Context interaction, illustrated in Figure 5, $F(15,360)=8.319, p<0.0005$. The CS $+*$ Placement interaction was not significant, $F(1,24)=1.921, p=0.178$, nor was no the Trial $* \mathrm{CS}+*$ Placement interaction, $F(15,360)=1.273, p=0.216$, the Context $* C S+*$ Placement interaction, $F<1$, the Trial $*$ Context $*$ CS + interaction, $F<1$, the Trial $*$ Context $*$ Placement interaction, $F<1$, or the Trial $*$ Context $*$ CS $+*$ Placement interaction, $F<1$. Although the Context * CS+ interaction did not reach significance, a further test was performed to follow it up. A grand difference score was computed for each rat, by subtracting the sum of all line crosses by that rat in the CS- from all line crosses by that rat in the CS+. A lower value therefore indicates a greater degree of locomotor activity in the CS- relative to the CS+, over the course of the experiment. Using the computed grand difference score, an independent-samples $t$ test was performed, with CS+ as the grouping variable (Figure 6). This test revealed that White rats had a significantly lower difference score (-83.25 +/- 7.88) than did Black rats $(-42.27+/-10.96)$. That is, White rats showed 
Figure 5. Locomotor activity, as indicated by line crosses, in each context during the 16 prestimulation test trials. All rats were tested in each context, in counterbalanced order. Data are averaged across both levels of Placement and CS+.

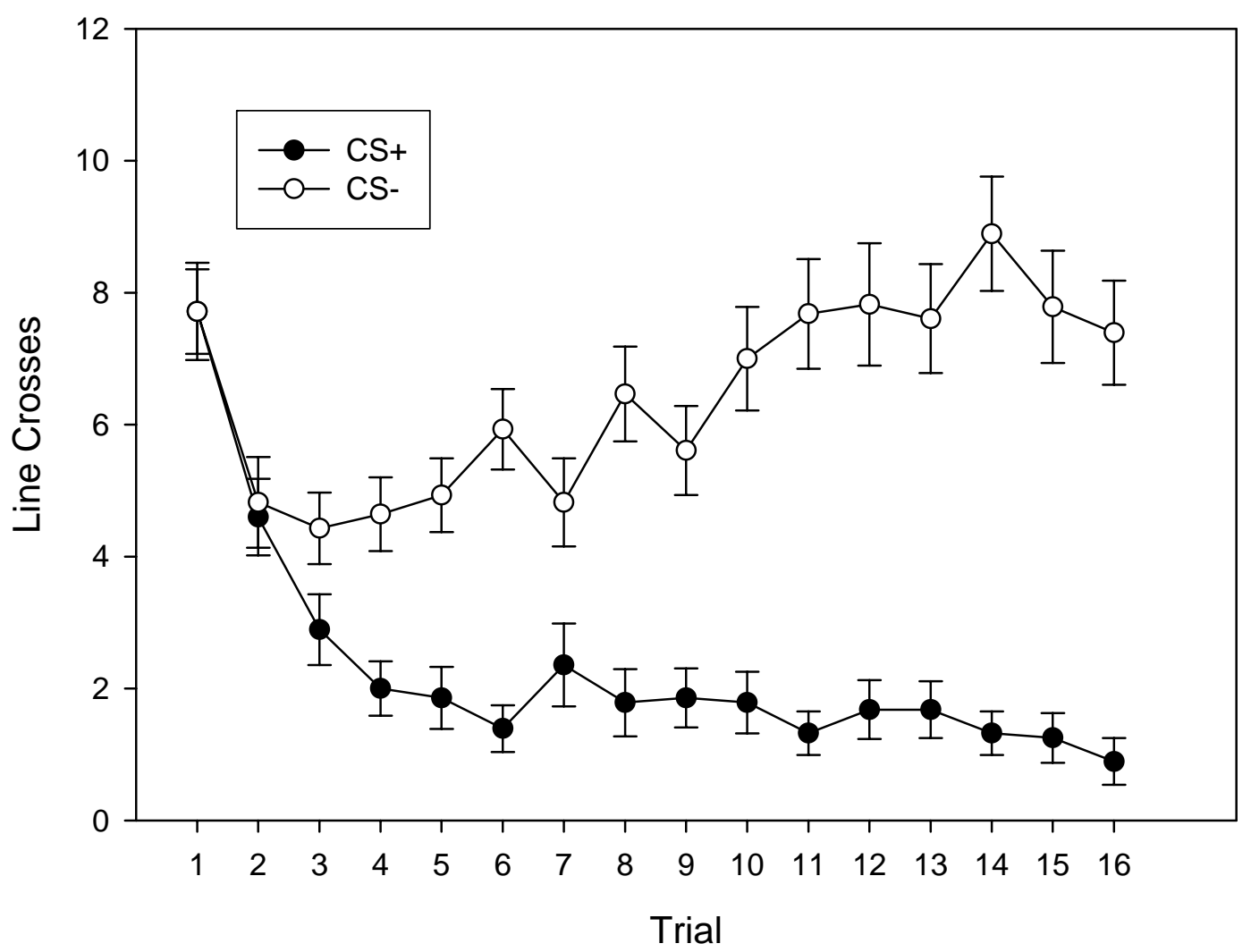


Figure 6. Locomotor activity grand difference scores, based on all prestimulation test trials, for rats in each of the CS+ assignment groups. The difference scores were calculated by subtracting the sum of all line crosses in the CS- from the sum of all line crosses in the CS+. Data are averaged across both levels of Placement. White rats showed a significantly larger difference in locomotor activity between their CS+ and CS- than did Black rats, $p=0.004$.

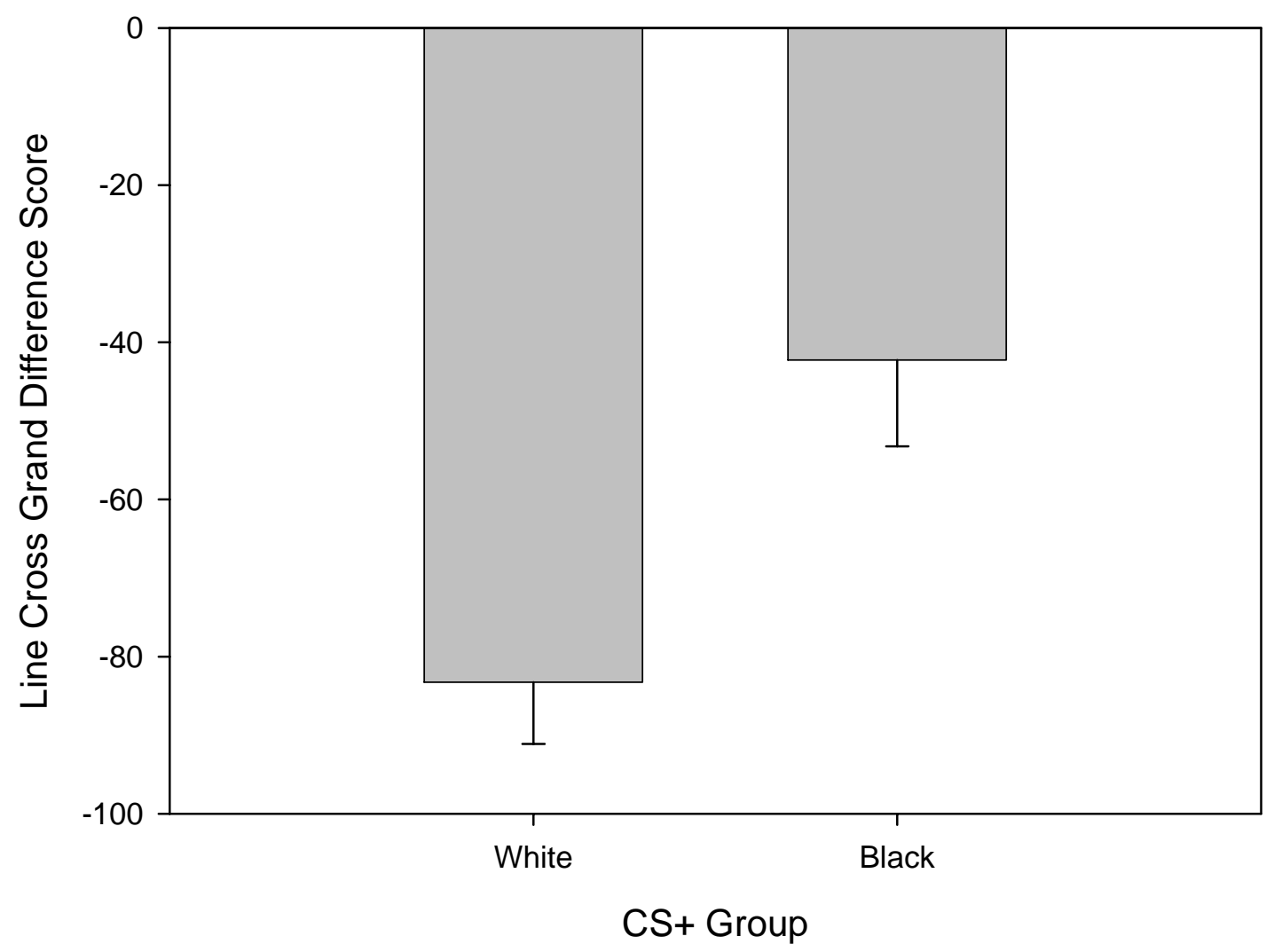


a greater degree of locomotor activity in the CS- relative to the CS+ than did black rats, $t(26)=-3.112, p=0.004$ (two-tailed).

To follow up the Trial * Context interaction, the 16 prestimulation test trials were grouped into four blocks of four consecutive trials each. A sum of line crosses in each context was then computed for each block. A difference score was then computed for each block, using the formula of line crosses in the CS+ minus line crosses in the CS-; a positive score therefore indicates more line crosses in the CS+ than in the CS- for that block. These difference scores are shown in Figure 7. Three planned repeated-measures ttests were computed, comparing the difference score from the first block to the difference scores from each of the other three blocks. A significant difference wherein the latter block's difference score was lower than the first block’s difference score therefore indicates a differential decrease in locomotor activity in the CS+ relative to the CS-. A Bonferroni correction requiring $\alpha / 3=0.05 / 3=0.0167$ was used. All $p$ values are reported uncorrected, and are one-tailed.

The second block had a significantly lower difference score $(-14.75+/-1.84)$ than the first block $(-4.40+/-2.00)$, with rats showing a decrease in locomotor activity in the CS+ relative to the CS- between the first and second blocks, $t(27)=5.089, p<0.00025$. There was a similar difference between the first and the third (-21.46 +/- 2.52) blocks, $t(27)=7.031, p<0.00025$. As well, the fourth block $(-26.54+/-3.06)$ had a significantly greater ratio of locomotor activity in the CS- to that in the CS+ than did the first block, $t(27)=7.755, p<0.00025$.

Rears. There was a significant main effect of Trial, indicating that there was at least one significant difference among the 16 trials, $F(15,360)=9.006, p<0.0005$. There 
Figure 7. Locomotor activity difference scores from each of the four blocks of prestimulation test trials. Data are averaged across both levels of Placement and CS+. Asterisk denotes a significant difference when compared to the difference score from Block 1.

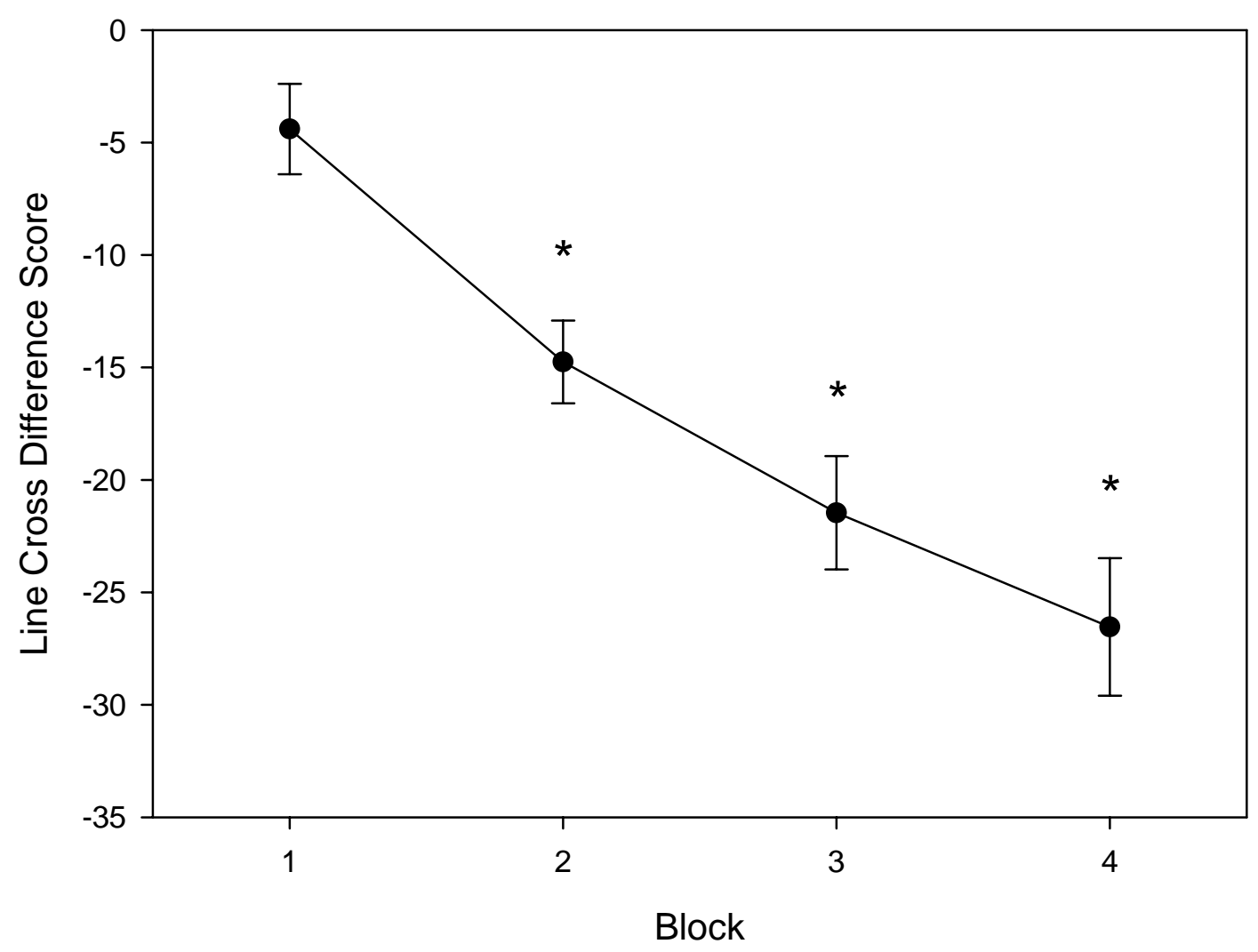


was a significant main effect of Context, with rats rearing fewer times in the CS+ (31.92 $+/-2.85)$ than in the CS- $(44.54+/-3.08), F(1,24)=13.589, p=0.001$. There was no significant main effect of CS+; thus White rats (75.05 +/- 6.60) did not differ significantly from Black rats (78.64 +/-9.04), $F<1$. As well, there was no significant main effect of Placement, in that BLA rats (78.44 +/- 5.74) did not differ significantly from Central Amygdala rats (71.50 +/- 12.05), $F<1$.

A number of interactions failed to reach statistical significance, including the Trial * CS+ interaction, $F(15,360)=1.450, p=0.122$; the Trial * Placement interaction, $F<1$, the Context $* C S+$ interaction, $F(1,24)=4.126, p=0.053$; and the Context * Placement interaction, $F<1$. The Trial * Context interaction was significant, $F(15,360)$ $=3.781, p<0.0005$. The CS $+*$ Placement interaction failed to reach statistical significance, $F(1,24)=1.180, p=0.288$, as did the Trial $*$ CS $+*$ Placement interaction, $F<1$; the Context $* \mathrm{CS}+*$ Placement interaction, $F<1$; the Trial $*$ Context ${ }^{*} \mathrm{CS}+$ interaction, $F(15,360)=1.893, p=0.023$; the Trial $*$ Context $*$ Placement interaction, $F$ $<1$; and the Trial $*$ Context $*$ CS $+*$ Placement interaction, $F(15,360)=1.069, p=$ 0.384 .

The Trial * Context * CS+ interaction, though not statistically significant at the $\alpha$ $=0.0167$ level, is taken as being sufficiently strong to warrant further analyses. This is partially because of its hierarchically superior position to the Trial * Context 2-way interaction. Two 16 (Trial) *2 (Context) *2 (Placement) mixed model ANOVAs were therefore conducted, one for each CS+ group. A Bonferroni correction of $\alpha / 2=0.05 / 2=$ 0.025 was used. All $p$ values are reported uncorrected. 
For the White group, Mauchly's Test of Sphericity revealed that the assumption of sphericity was violated for Trial, $\chi(119)=266.214, p<0.0005$. The Huynh-Feldt $\varepsilon$ correction was therefore used, $\varepsilon=0.741$. As well, the assumption of sphericity was violated for the Trial * Context interaction term, $\chi(119)=161.551, p=0.028$. Again, the Huynh-Feldt $\varepsilon$ correction was used, $\varepsilon=0.880$. There was a significant main effect of Trial, indicating that there was at least one significant difference among the 16 trials, $F(11.121,166.820)=6.513, p<0.0005$. There was also a significant main effect of Context, indicating that there were significantly fewer rears in the CS+ $(28.58+/-3.07)$ than in the CS- $(46.47+/-4.12), F(1,15)=24.449, p<0.0005$. There was no significant effect of Placement, indicating that White BLA rats did not exhibit a number of rears (80.07 +/ 8.31) significantly different from White Central Amygdala rats (63.00 +/9.22), $F(1,15)=1.427, p=0.251$. There was no significant Trial $*$ Placement interaction, $F<1$. As well, there was no significant Context * Placement interaction, $F<1$. There was a significant Trial * Context interaction (Figure 8), $F(13.195,197.925)=2.815, p=$ 0.001. The Trial * Context * Placement interaction was not significant, $F<1$.

To follow up the Trial * Context interaction, the 16 prestimulation test trials were grouped into four blocks of four consecutive trials each. A sum of rears in each context was then computed for each block. A difference score was then computed for each block, using the formula of rears in the CS+ minus rears in the CS-; a positive score therefore indicates more rears in the CS+ than in the CS- for that block. These difference scores are shown in Figure 9. Three unplanned repeated-measures $t$-tests were computed, comparing the difference score from the first block to the difference scores from each of the other 
Figure 8 . Rears by White rats in each context during the 16 prestimulation test trials. All rats were tested in each context, in counterbalanced order. Data are averaged across both levels of Placement.

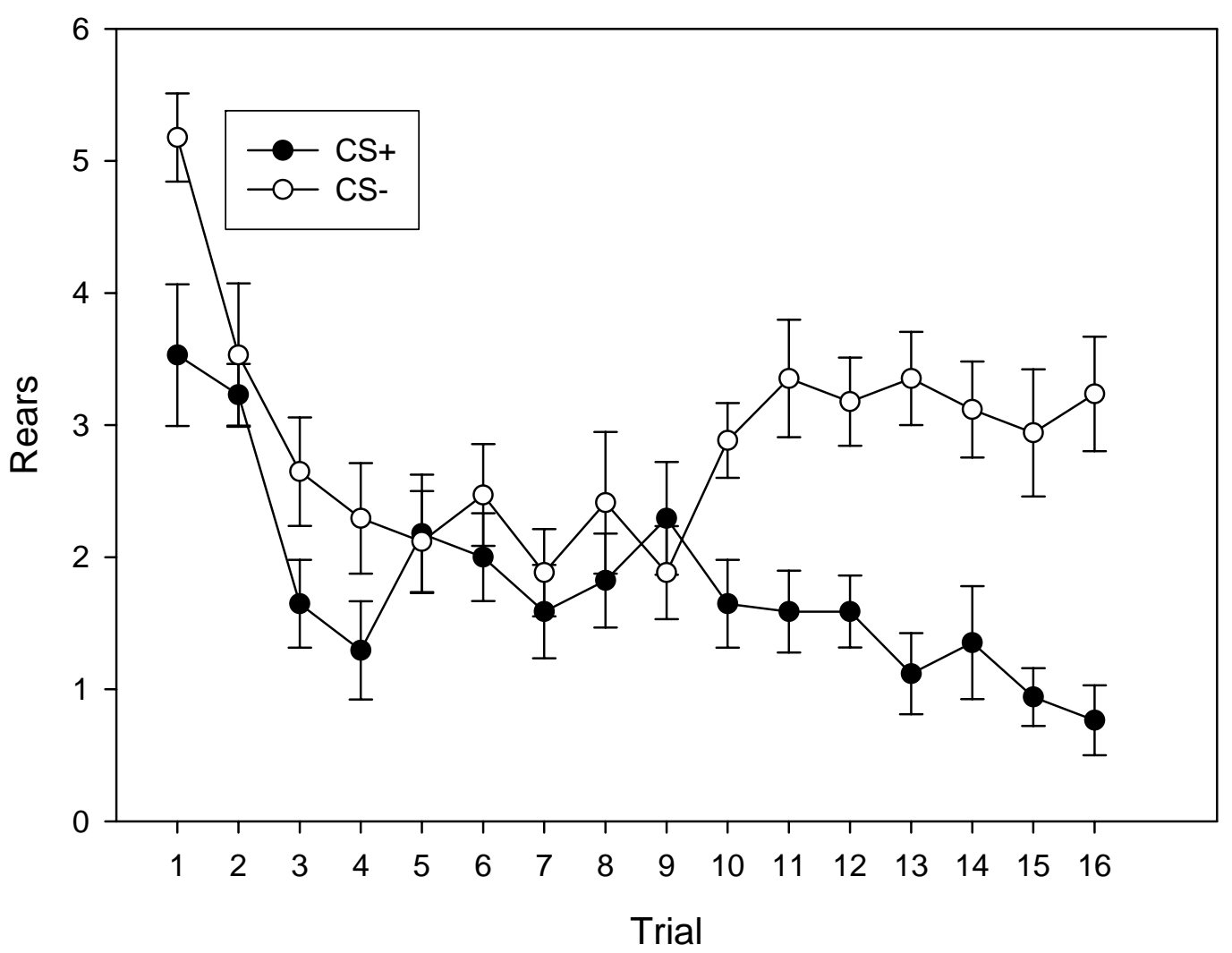


Figure 9. Rearing difference scores from the White group, from each of the four blocks of prestimulation test trials. Data are averaged across both levels of Placement and CS+. Each of the latter three blocks was compared with the first block. No significant differences were found at $\alpha=0.0167$. However, the difference of the fourth block from the first block approached statistical significance, $p=0.025$ (uncorrected).

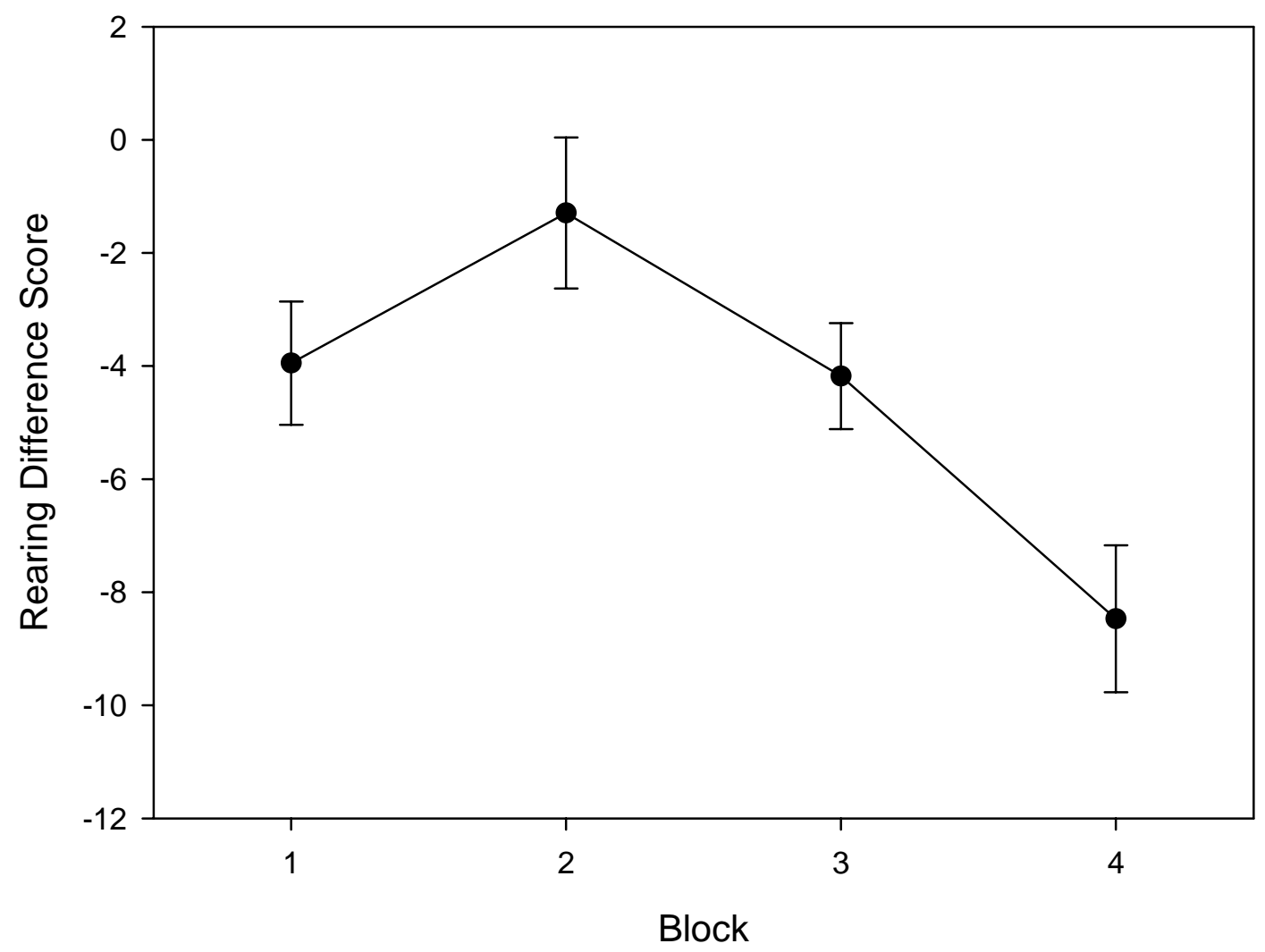


three blocks. A significant difference wherein the latter block's difference score was lower than the first block's difference score therefore indicates a differential decrease in rearing in the CS + relative to the CS-. A Bonferroni correction requiring $\alpha / 3=0.05 / 3=$ 0.0167 was used. All $p$ values are reported uncorrected, and are two-tailed. There was no significant difference between the difference scores from the first block (-3.95 +/- 1.09) and the second block $(-1.29+/-1.34), t(16)=-1.737, p=0.102$. There was also no significant difference between the first block and the third block $(-4.18+/-0.94), t(16)=$ $0.159, p=0.875$. However, the difference between the first and fourth blocks approached statistical significance; rats showed a higher ratio of rears in the CS- relative to the CS+ in the fourth block than in the first block, $t(16)=2.465, p=0.025$.

For the Black group there was a significant main effect of Trial, indicating that there was at least one significant difference among the 16 trials, $F(15,135)=4.284, p<$ 0.0005. There was no significant main effect of Context; averaged across all levels of the other factors, there was no difference in rearing between the CS+ $(37.09+/-5.31)$ and the CS- (41.54 +/- 4.67), $F<1$. There was also no significant main effect of Placement, indicating that Black BLA rats $(76.00$ +/ 7.71) did not differ from Black Central Amygdala rats (85.67 +/- 30.17) in the total number of rears, $F<1$. There was no significant Trial * Placement interaction, $F<1$. There was also no significant Context * Placement interaction, $F<1$. There was a significant Trial * Context interaction (Figure 10), $F(15,135)=2.896, p=0.001$. The Trial $*$ Context $*$ Placement interaction was not significant, $F(15,135)=1.148, p=0.321$.

The Trial * Context interaction in the Black ANOVA was followed-up with the same method as was used to follow up the Trial * Context interaction in the White 
Figure 10. Rears by Black rats in each context during the 16 prestimulation test trials. All rats were tested in each context, in counterbalanced order. Data are averaged across both levels of Placement.

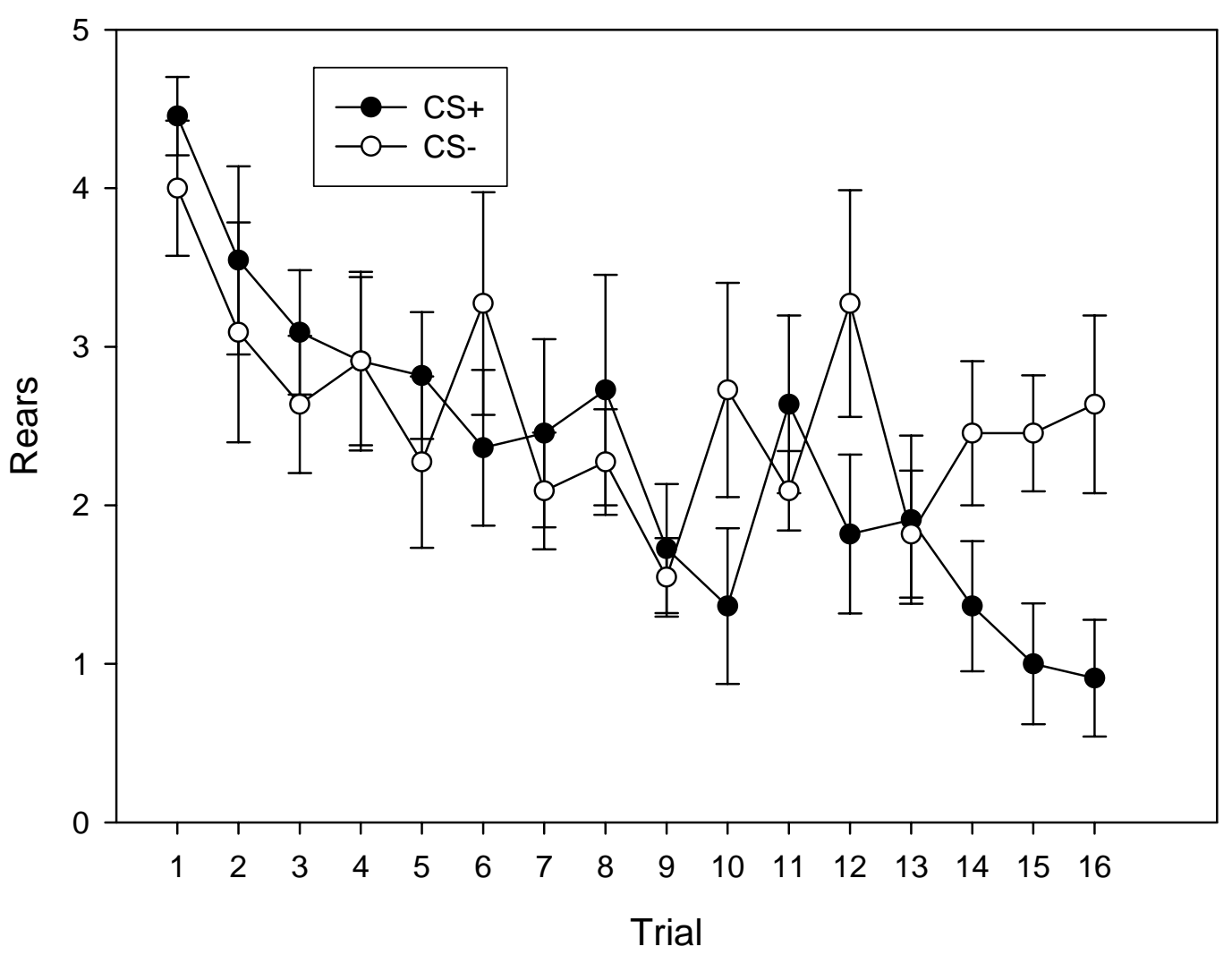


ANOVA, by using difference scores in four blocks. Figure 11 illustrates these difference scores. There was no significant difference between the difference scores from the first block (1.36 +/- 0.80) and the second block $(0.45+/-1.10), t(10)=0.752, p=0.470$. The difference between the difference scores from the third block $(-2.09+/$ - 1.76) and the first block was not significant, $p=0.075$. The difference score from the fourth block $(-4.18+/-$ 1.72), however, was significantly lower than that from the first block; Black rats showed a significantly greater ratio of rears in the CS- relative to the CS+ in the fourth block than in the first block, $t(10)=3.170, p=0.010$.

CPP Test

CPP test results were analyzed using a 2 (context) $* 2(\mathrm{CS}+) * 2$ (placement) mixed model ANOVA. The measure used was the percentage of time spent in either context. As seen in Figure 12, there was a significant main effect of context; averaging across groups, rats spent a significantly greater proportion of time in the CS- (77.6\%) than in the CS $+(22.4 \%), F(1,24)=69.320, p<0.0005$. There was no significant main effect of placement, attributable to the fact that such a test averages across contexts, giving two means of exactly 50 with no variance, $F<1$. For the same reason, there was so significant main effect of CS+, $F<1$. The Context * Placement interaction term tests whether the effect of context is the same across the two placements; that is, it tests whether the groups differed in their relative preferences. This test did not indicate a significant difference in the degree of CPP between the BLA group (75.8\% in CS-) and the Central Amygdala group (82.2\% in CS-), $F(1,24)=0.685, p=0.416$. The CS $+*$ Context interaction term tests whether the rats differed in their CPP according to their 
Figure 11. Rearing difference scores from the Black group, from each of the four blocks of prestimulation test trials. Data are averaged across both levels of Placement and CS+. Asterisk denotes a significant difference when compared to the difference score from Block 1.

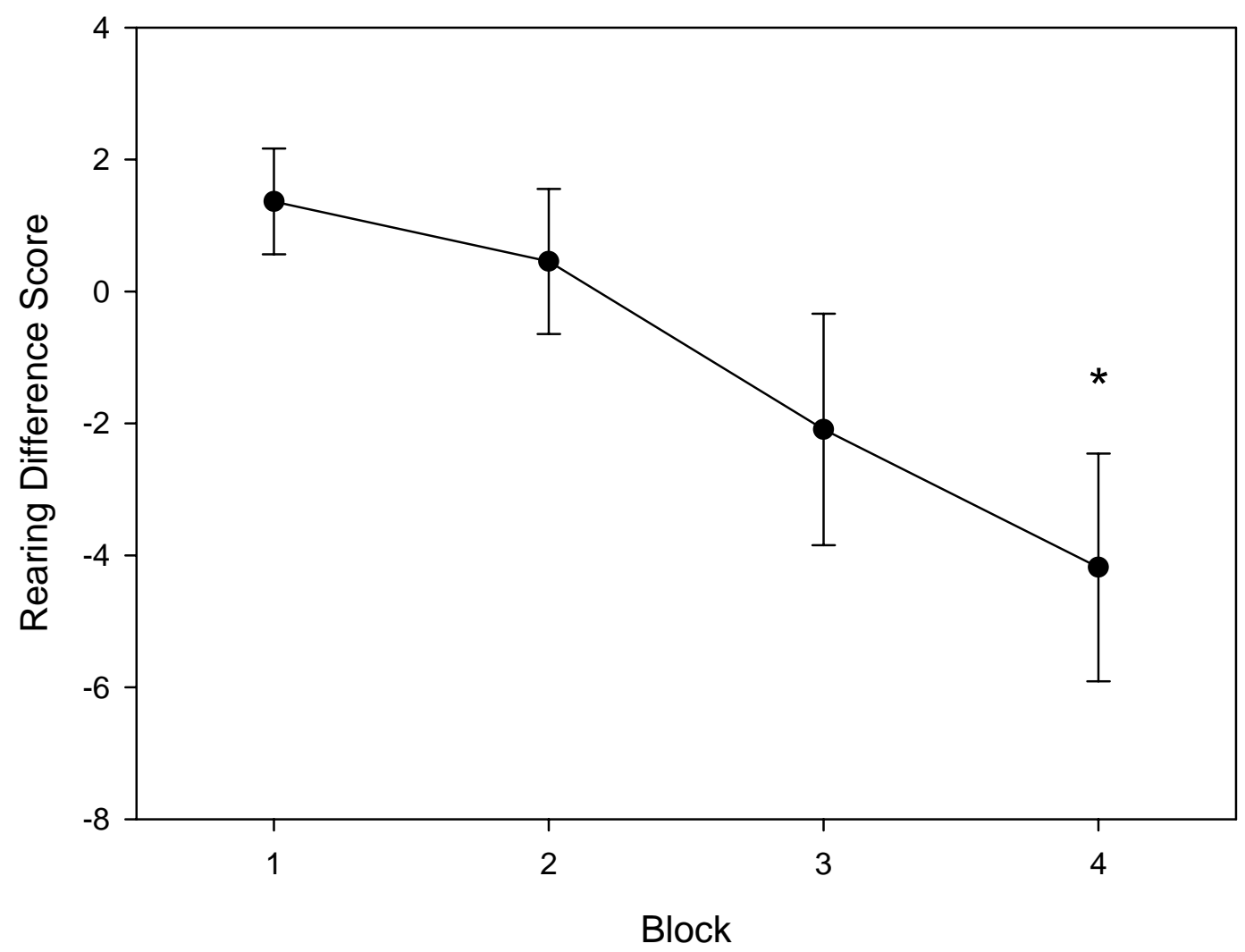


Figure 12. Percentage of time, spent by the rats, in each context during the five-minute conditioned place preference test. Data are averaged across all groups. Rats spent significantly less time in the CS- than in the CS+, $p<0.0005$.

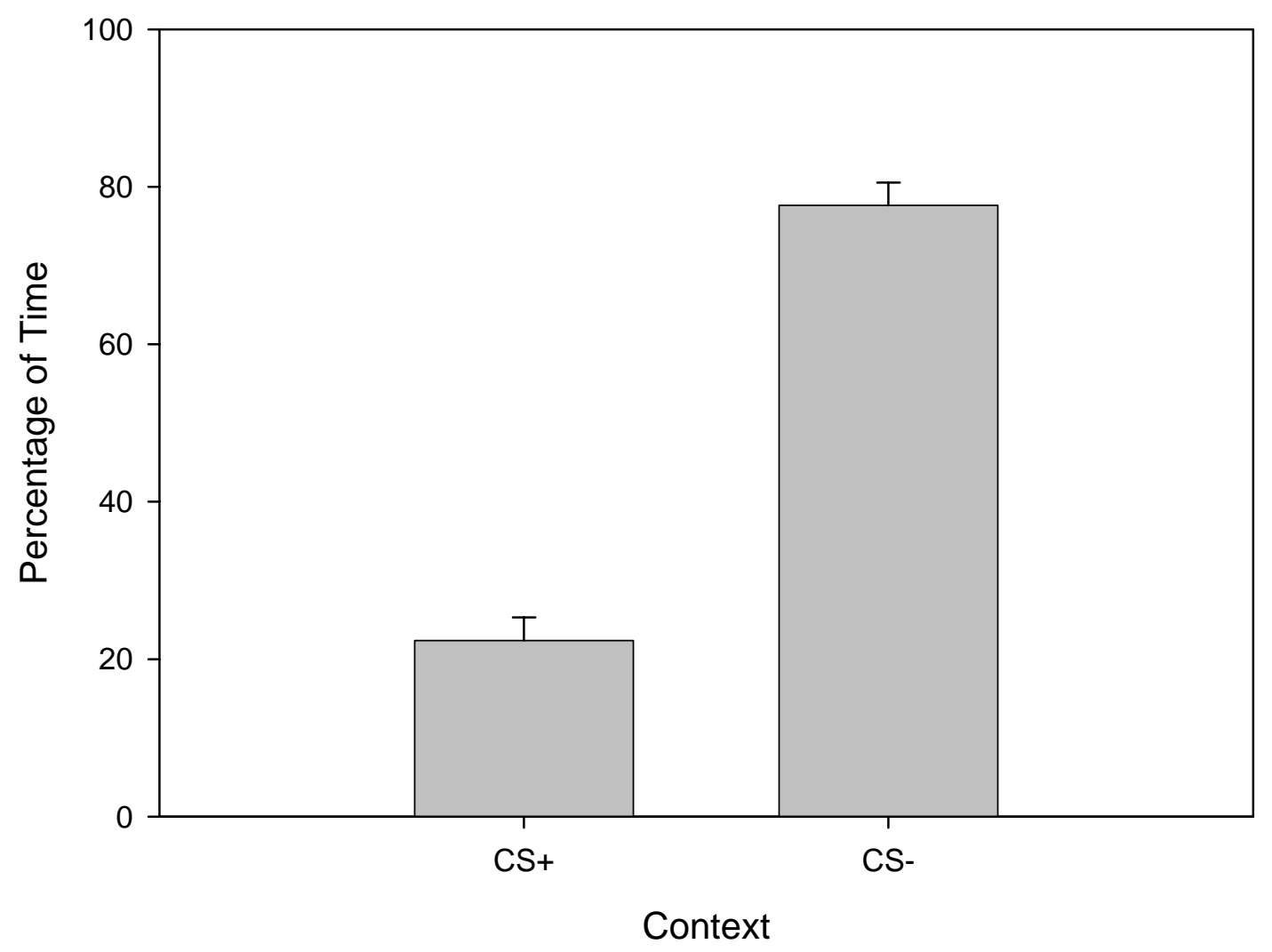


assignment to CS+ condition. There was no significant CS + * Context interaction; assignment to the CS+ conditions did not affect CPP, $F<1$. The CS $+*$ Placement interaction term is meaningless, because it averages across the two contexts, which sum to exactly $100 \%$ for each animal. This interaction was therefore not significant, $F<1$. There was no significant Context $* \mathrm{CS}+*$ Placement interaction, $F<1$.

\section{Between-Subjects Switch Test}

Data from the switch test were analyzed using 2 (Context) $* 2(\mathrm{CS}+) * 2$ (Placement) univariate ANOVAs. Dependent variables were: stage of convulsion (Stage), latency to forelimb clonus (Latency), Clonus Duration, AD Duration, number of falls (Falls), and wet-dog shakes (WDS). To correct for the increased probability of Type I error resulting from multiple analyses, a Bonferroni correction was used; the corrected $\alpha$ level was $0.05 / 6=0.00833$. All $\alpha$ levels are reported uncorrected. There were no significant effects found in this test.

Stage. The omnibus ANOVA analyzing convulsion stage results did not reach significance; there were no significant main effects or interactions, $F(6,21)=1.512, p=$ 0.223 .

Latency. One rat was excluded from the analysis of Latency data because it did not display a Stage 3 or greater convulsion in the trial of interest. The omnibus ANOVA using Latency data did not reach significance; there were no significant main effects or interactions, $F(6,20)=1.961, p=0.120$.

Clonus duration. The omnibus ANOVA analyzing Clonus Duration results did not reach significance; no main effects or interactions were found, $F<1$. 
$A D$ duration. The omnibus ANOVA for AD duration did not reach significance; no main effects or interactions were found, $F(6,21)=1.418, p=0.254$.

Falls. The omnibus ANOVA analyzing Falls did not reach significance; there were no significant main effects or interactions, $F<1$.

WDS. The omnibus ANOVA analyzing WDS results did not reach significance; there were no significant main effects or interactions, $F<1$. Within-Subjects Switch Test

Data from the switch test were analyzed using 2 (Context) $* 2(\mathrm{CS}+) * 2$ (Placement) mixed model ANOVAs. Dependent variables were: stage of convulsion (Stage), latency to forelimb clonus (Latency), Clonus Duration, AD Duration, number of falls (Falls), and wet-dog shakes (WDS). To correct for the increased probability of Type I error resulting from multiple analyses, a Bonferroni correction was used; the corrected $\alpha$ level was $0.05 / 6=0.00833$. All $\alpha$ levels are reported uncorrected. There were no significant effects found in this test.

Stage. There were no significant main effects or interactions found in the ANOVA analyzing Stage. As shown in Figure 13, there was no significant main effect of Context, indicating that convulsion stages measured in the CS+ $(5.39+/-0.17)$ did not differ significantly from those measured in the CS- $(5.00,+/-0.25), F<1$. There was no significant main effect of Placement, indicating that BLA rats $(5.15+/-0.20)$ did not differ significantly from Central Amygdala rats (5.31+/- 0.25), $F<1$. There was no significant main effect of CS+, indicating that rats in the White group (5.09+/- 0.24) did not differ significantly from rats in the Black group (5.36 +/- 0.17), $F<1$. 
Figure 13. Convulsion stage, as observed in each context in the Within-Subjects Switch Test. All rats were tested in each context, in counterbalanced order. Data are averaged across groups. The difference between stage observed in the CS+ and in the CS- was not significant, $p=0.727$ (uncorrected).

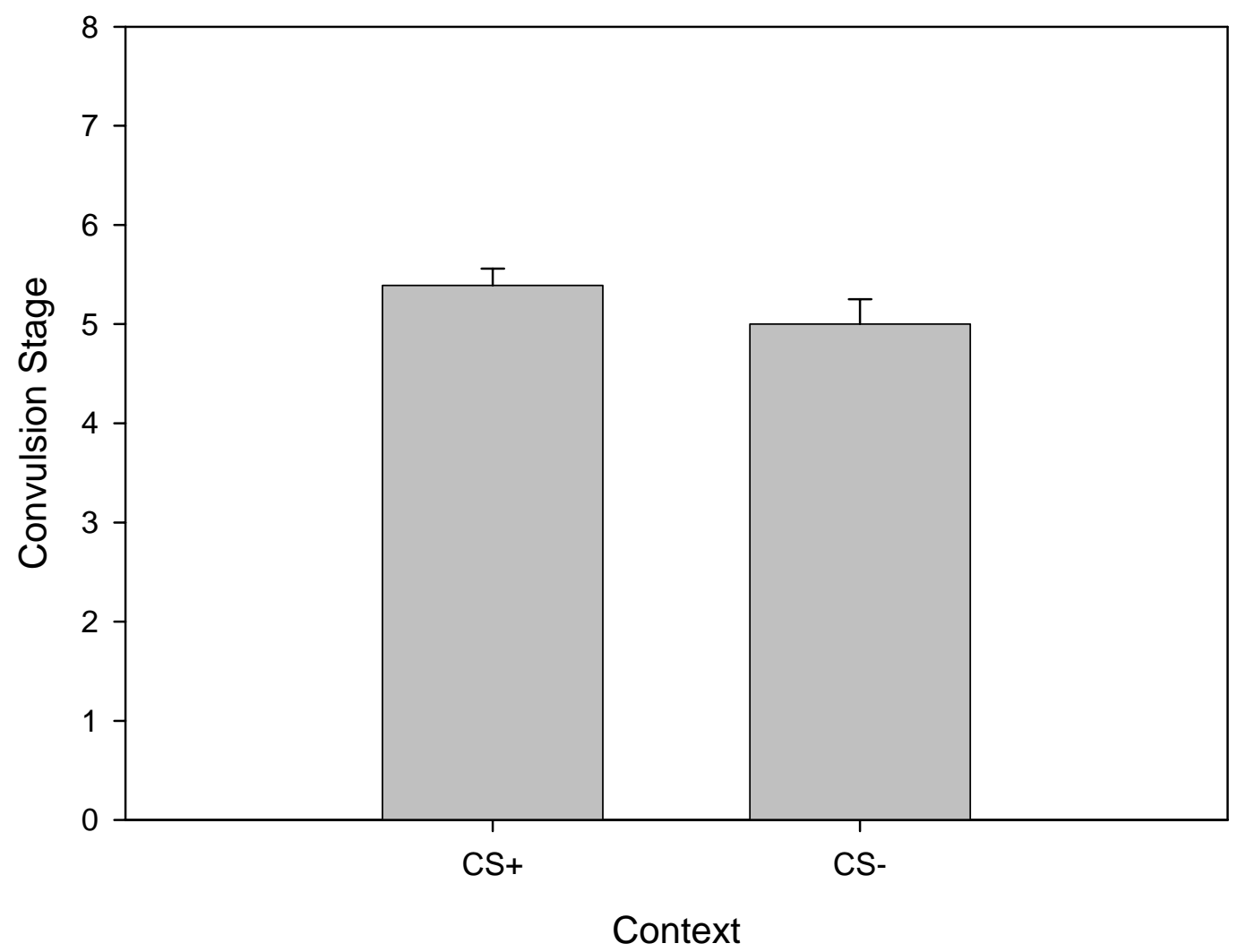


There was no significant Placement $*$ CS+ interaction, $F<1$. There was no significant Context * Placement interaction, $F(1,24)=1.177, p=0.289$. There was no significant Context * CS + interaction, $F(1,24)=3.205, p=0.086$. There was no significant Context * Placement * CS+ interaction, $F<1$.

Latency. One rat was excluded from the analysis of Latency data because it did not display a Stage 3 or greater convulsion in one of its Switch Test trials; latency could therefore not be measured in that trial. The ANOVA analyzing Latency data did not yield any significant main effects or interactions. As shown in Figure 14, there was no significant main effect of Context; rats showed similar latencies in the CS+ (5.04 +/- 1.19 s) and in the CS- $(5.26+/-1.25 \mathrm{~s}), F<1$.

There was no significant Placement $* \mathrm{CS}+$ interaction, $\mathrm{F}<1$. There was no significant Context * Placement interaction, $F<1$. There was no significant Context * CS+ interaction, $F<1$. There was no significant Context * Placement * CS+ interaction, $F<1$.

Clonus duration. No significant main effects or interactions were found in the ANOVA analyzing Clonus Duration results. As shown in Figure 15, there was no significant main effect of Context, indicating that clonus durations were similar in the CS+ (36.25 +/- 3.67 s) and the CS- (34.18+/- 3.70 s), $F<1$. There was no significant main effect of Placement, indicating that rats with BLA electrodes had durations of clonus (34.88 +/- 2.96 s) similar to those of rats with Central Amygdala electrodes (36.06 $+/-4.22 \mathrm{~s}), F<1$. There was no significant main effect of CS+, indicating that rats in the White group (33.26 +/-3.42 s) did not differ significantly from those in the Black group $(38.23+/-3.01 \mathrm{~s}), F(1,24)=1.048, p=0.316$. 
Figure 14. Latency to a stage 3 or greater convulsion, as measured in each context in the Within-Subjects Switch Test. All rats were tested in each context, in counterbalanced order. Data are averaged across groups. The difference between latencies measured in the CS+ and in the CS- was not significant, $p=0.917$ (uncorrected).

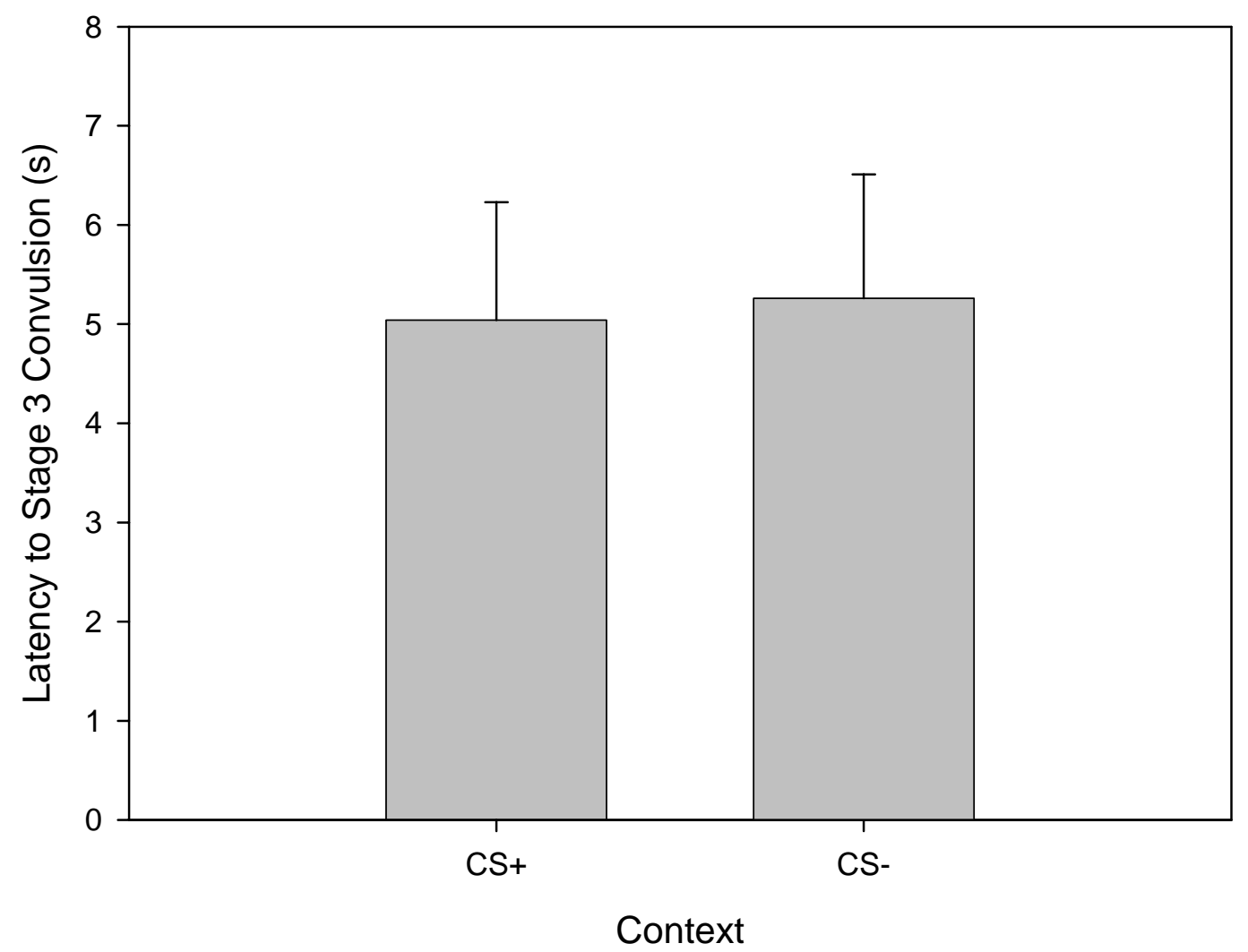


Figure 15. Clonus duration, as measured in each context in the Within-Subjects Switch Test. All rats were tested in each context, in counterbalanced order. Data are averaged across groups. The difference between clonus duration in the CS+ and in the CS- was not significant, $p=0.650$ (uncorrected).

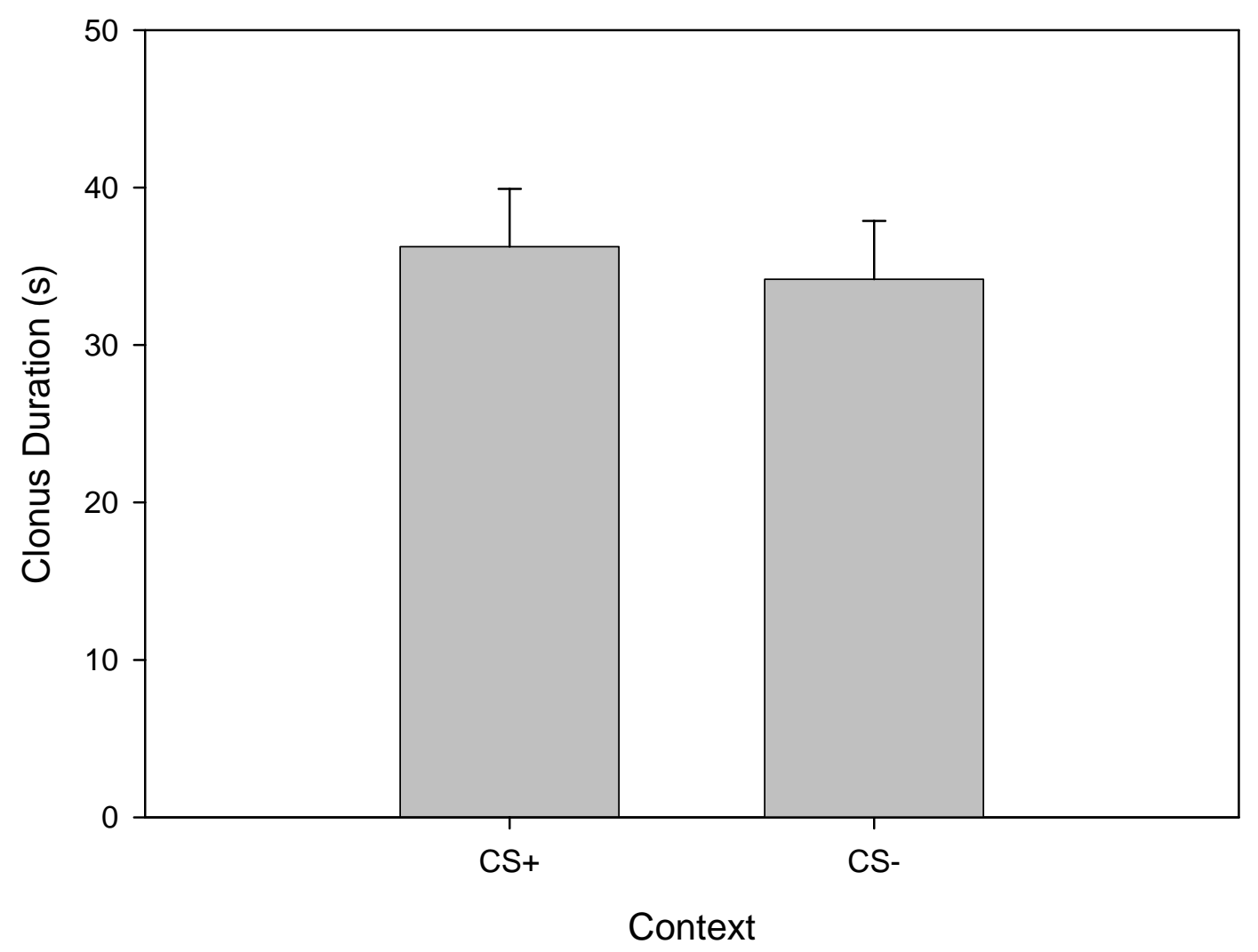


There was no significant Placement $*$ CS + interaction, $F<1$. There was no significant Context $*$ Placement interaction, $F(1,24)=1.699, p=0.205$. There was no significant Context $*$ CS + interaction, $F(1,24)=1.639, p=0.213$. There was no significant Context * Placement * CS+ interaction, $F<1$.

$A D$ duration. The ANOVA analyzing AD Duration results yielded no significant main effects or interactions. As shown in Figure 16, there was no significant effect of Context, indicating that rats had similarly long seizures in the CS+ $(72.25+/-6.30 \mathrm{~s})$ and the CS- (68.07+/- $6.82 \mathrm{~s}), F<1$. There was no significant main effect of Placement, indicating that rats with electrodes in the BLA had long seizures $(66.58+/-6.20$ s) similar to those of rats with electrodes in the central nucleus of the amygdala (79.12 +/- $11.69 \mathrm{~s})$, $F<1$. There was no significant main effect of CS+, indicating that rats in the White group had long seizures (66.26 +/- 7.55 s) similar to those of rats in the Black group (76.18+/- $7.97 \mathrm{~s}), F<1$.

There was no significant Placement * CS+ interaction, $F(1,24)=1.628, p=$ 0.214. There was no significant Context * Placement interaction; $F<1$. There was no significant Context $*$ CS + interaction, $F<1$. There was no significant Context * Placement $*$ CS + interaction, $F<1$.

Falls. The ANOVA analyzing Falls results yielded no significant main effects or interactions. As shown in Figure 17, there was no significant main effect of Context, indicating that rats had similar numbers of falls in the CS+ $(2.68+/-0.44)$ as in the CS(1.93 +/- 0.36), $F<1$. There was no significant main effect of Placement, indicating that BLA rats (2.28 +/- 0.42) did not differ from Central Amygdala rats (2.38+/- 0.64), $F<1$. 
Figure 16. AD duration as measured in each context in the Within-Subjects Switch Test. All rats were tested in each context, in counterbalanced order. Data are averaged across groups. The difference between AD durations measured in each context was not significant, $p=0.800$ (uncorrected).

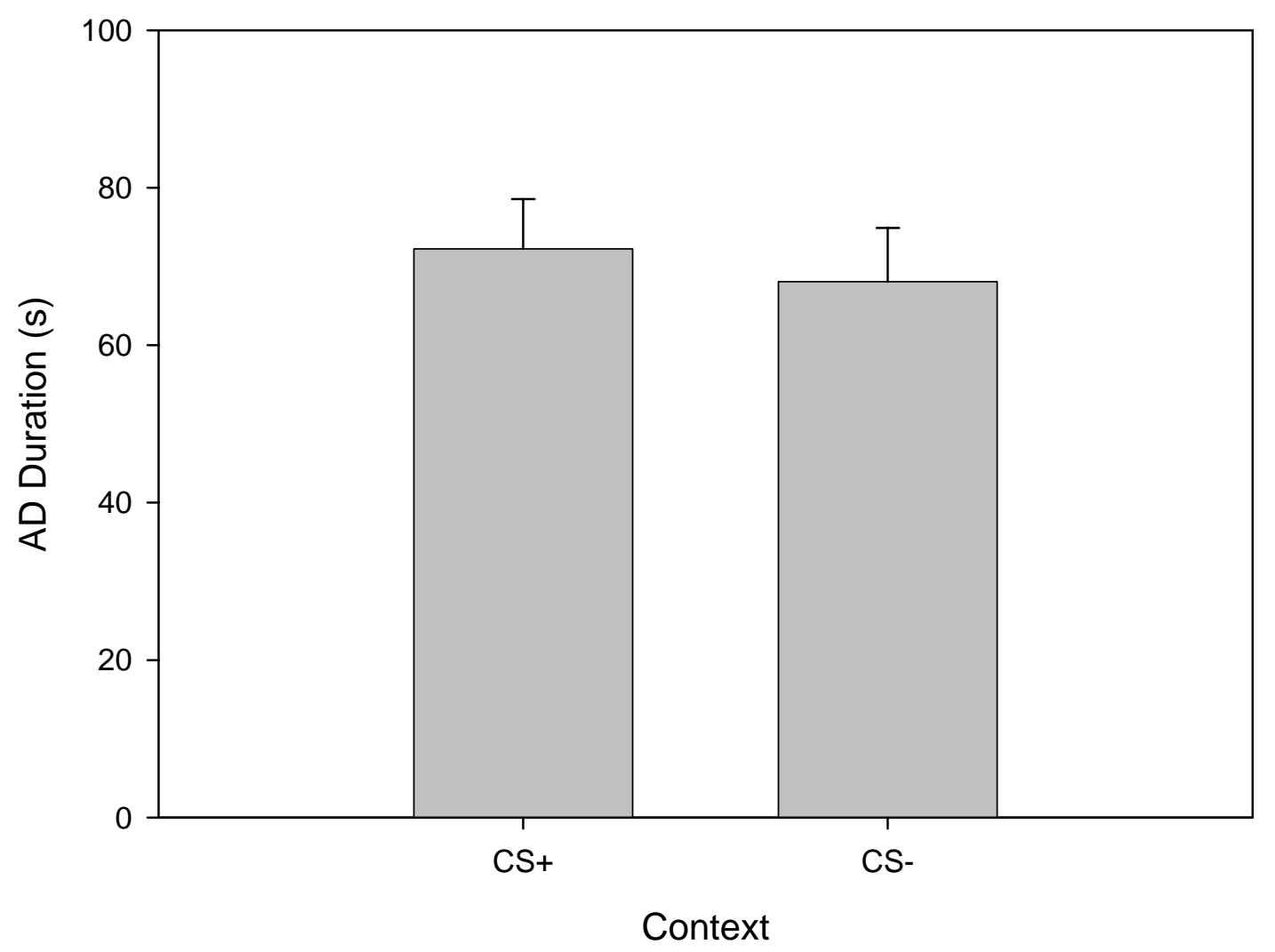


Figure 17. Falls by rats, as observed in each context in the Within-Subjects Switch Test. All rats were tested in each context, in counterbalanced order. Data are averaged across groups. The difference between falls observed in the CS+ and in the CS- was not significant, $p=0.419$ (uncorrected).

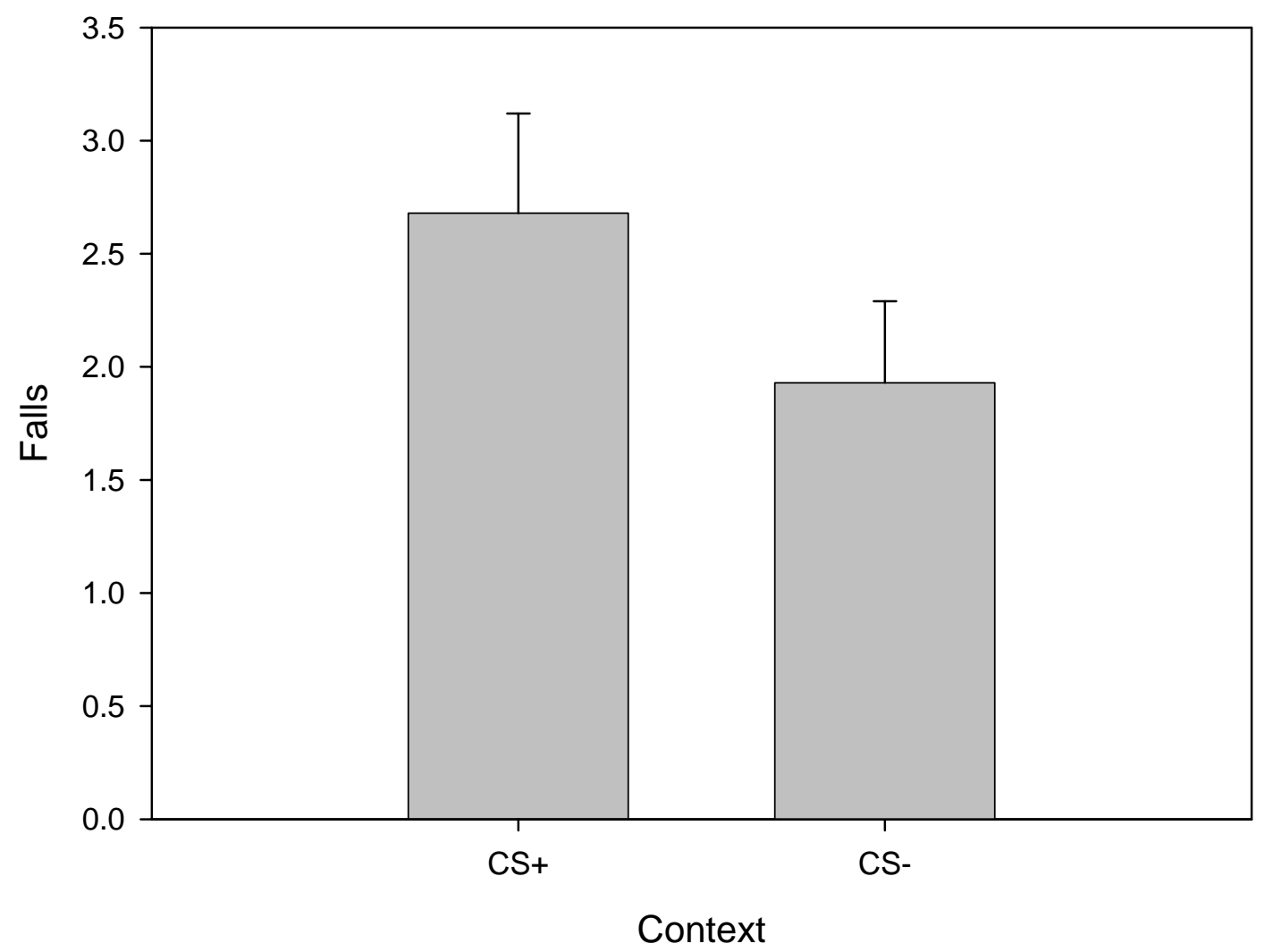


There was no significant main effect of CS+, indicating that rats in the White group (2.24 +/- 0.43) did not differ from rats in the Black group (2.41+/- 0.59), $F<1$.

There was no significant Placement * CS+ interaction, $F<1$. There was no significant Context * Placement interaction, $F(1,24)=1.577, p=0.221$. There was no significant Context $*$ CS + interaction, $F(1,24)=1.861, p=0.185$. There was no significant Context * Placement $*$ CS+ interaction, $F<1$.

WDS. In the ANOVA analyzing WDS data, here were no significant main effects or interactions. As shown in Figure 18, there was no significant main effect of Context, indicating that the number of WDSs measured in the CS+ $(2.21+/-0.72)$ did not differ significantly from that measured in the CS- $(0.75+/-0.26), F(1,24)=3.959, p=0.058$. There was no significant main effect of Placement, indicating that BLA rats $(1.48+/$ 0.43) did not differ significantly from Central Amygdala rats $(1.50+/-0.95), F<1$. There was no significant main effect of CS+, indicating that rats in the White group $(1.47+/-$ 0.52) did not differ significantly from rats in the Black group (1.50 +/- 0.66), $F<1$.

There was no significant Placement $*$ CS+ interaction, $F(1,24)=1.89, p=0.181$. There was no significant Context * Placement interaction, $F<1$. There was no significant Context $*$ CS + interaction, $F<1$. There was no significant Context * Placement $*$ CS+ interaction, $F(1,24)=1.435, p=0.243$.

ADT Switch Test

Using the ADT measured in each context as the dependent variable, a 2 (Context) * $2(\mathrm{CS}+) * 2$ (Placement) mixed model ANOVA was computed. One rat, belonging to the BLA and White groups, was excluded from this analysis because the EEG recorded from its electrodes was too noisy for accurate identification of AD. As shown in Figure 
Figure 18. Wet dog shakes (WDSs) by rats, as observed in each context in the WithinSubjects Switch Test. All rats were tested in each context, in counterbalanced order. Data are averaged across groups. The difference between WDSs measured in the CS+ and those in the CS- was not significant, $p=0.058$ (uncorrected).

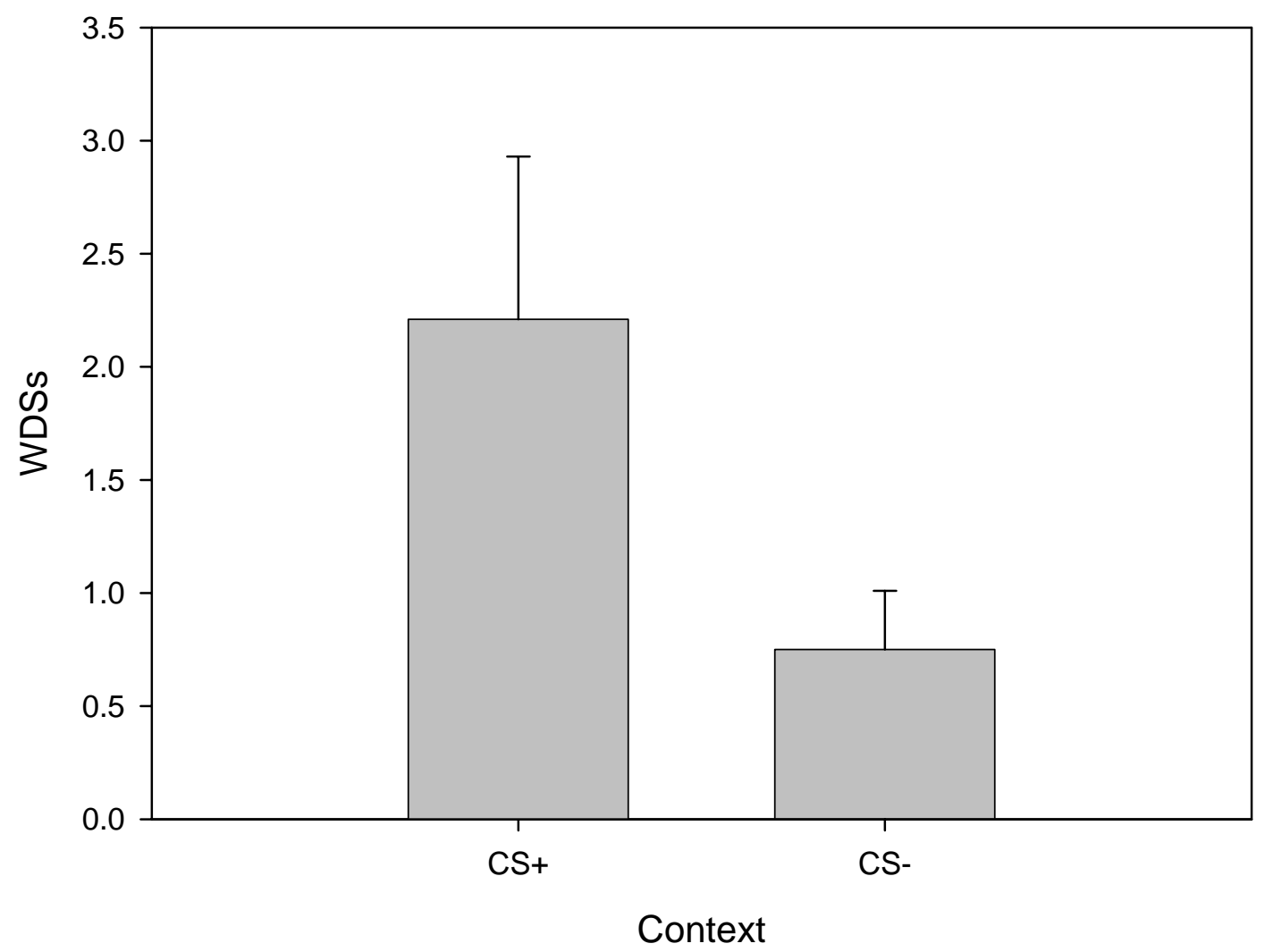


19, there was no significant main effect of Context; rats displayed similar ADTs in the $\mathrm{CS}+(114.44+/-13.41 \mu \mathrm{A})$ and the CS- $(113.33+/-10.24 \mu \mathrm{A}), F<1$. There was no significant main effect of CS+, indicating that rats assigned to the White group had ADTs $(109.69+/-12.60 \mu \mathrm{A})$ similar to those of the Black group $(120.00+/-19.85 \mu \mathrm{A})$. As seen in Figure 20, there was no significant main effect of placement, indicating that rats with electrodes in the central nucleus of the amygdala did not have significantly different ADTs $(85.62+/-6.44 \mu \mathrm{A})$ from those of rats with electrodes in the BLA $(125.79+/$ $14.38 \mu \mathrm{A}), F(1,23)=2.655, p=0.117$.

There was no significant Context * CS+ interaction; the effect of Context did not vary according to assignment to $\mathrm{CS}+, F<1$. There was no significant Context * Placement interaction, indicating that the effect of Context did not vary according to electrode placement, $F<1$. There was no significant $C S+*$ Placement interaction, indicating that the effect of electrode placements did not vary according to assignment to $\mathrm{CS}+, F<1$. There was no significant Context $* \mathrm{CS}+*$ Placement interaction, $F<1$.

\section{Discussion}

In the present experiment, seizures were kindled in rats by electrical stimulation of the amygdala in one context (CS+), and the rats were connected to the equipment but not stimulated in another context (CS-). Electrode tips were located in either the basolateral amygdala (BLA) or the central nucleus of the amygdala (Central Amygdala), and there were no differences in kindling rate, conditioned effects, or afterdischarge threshold between those two groups. The rats developed conditioned anticipatory defensive responses in the CS+; in that environment, they froze more, displayed reduced locomotor activity, and reared less, relative to the CS-. These differences were enhanced 
Figure 19. ADT as measured in each context, at the end of the experiment. Each rat was tested in each context, one context per day, over two days in counterbalanced order. There was no significant difference between ADTs measured in the two contexts.

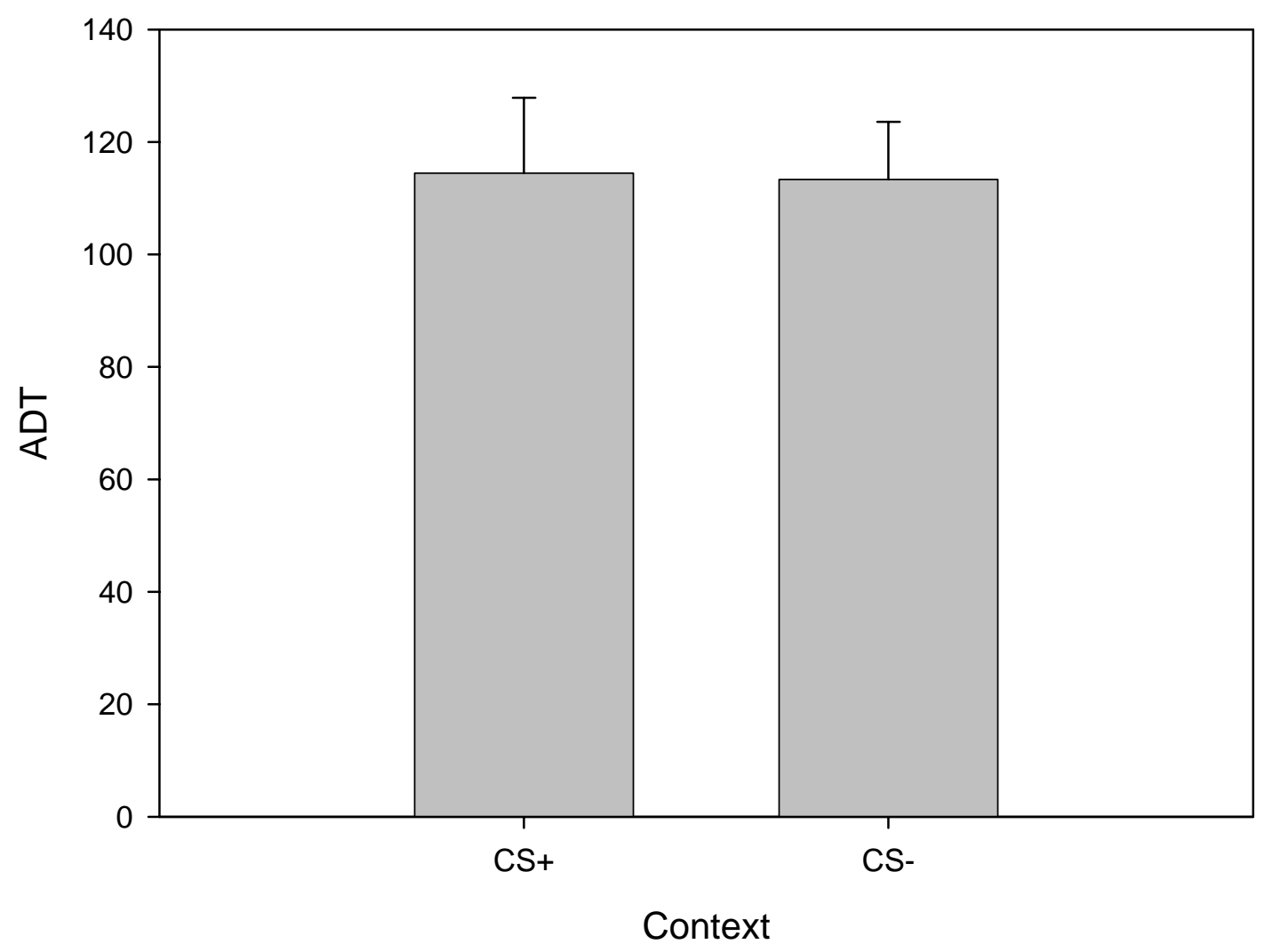


Figure 20. Mean ADT, as measured in both contexts, for BLA and Central Amygdala rats. The difference between the two groups was not significant, $p=0.117$, uncorrected.

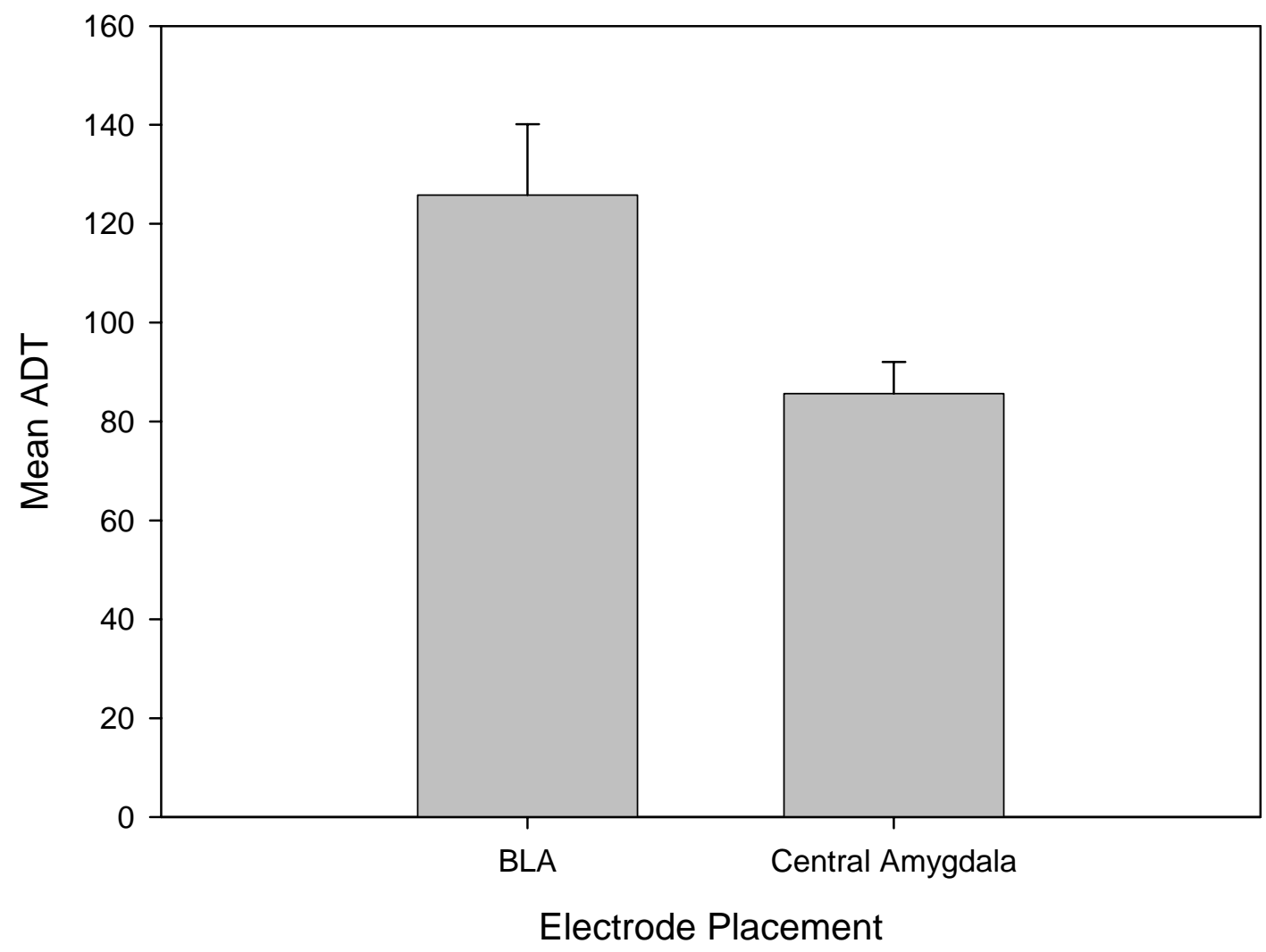


by the White context, in that rats kindled in the White context showed the greatest differences between the two contexts. There was no difference in the rate of kindling between rats kindled in the White context and those kindled in the Black context. The rats showed a conditioned place aversion for the CS+, indicating that amygdaloid kindling, or some aspect thereof, is punishing. I failed, however, to find contextually conditioned effects on convulsions, seizures, or afterdischarge threshold, using switch tests in which rats were stimulated in each context. Because I found conditioned effects on interictal behaviours but not on ictal measures, my results are therefore a partial replication of the results of Barnes et al. (2001).

My results clearly indicate that the rats were able to form learned associations between the contexts and the events that occurred therein, including kindling. Given the results of Paredes et al. (2000), who used a place conditioning procedure to show that the aftereffect of generalized amygdaloid seizures can be rewarding, this suggests that the rats attended to the ictal events. That is, rats in the present experiment experienced both kindled seizures and their aftereffects in the CS+. A conditioned aversive response therefore seems to indicate that some aversive aspect of the ictal experience was more salient than the rewarding aftereffect of the seizures.

Implicit in the argument above is the idea that some aspect of amygdaloid kindling is aversive. But there are many aspects of kindling, and it is not immediately clear which is aversive. Below I consider several possibilities.

It is possible, for example, that the 1-sec train of electrical stimulation used to elicit a seizure is aversive. Using chemical stimulation of the BLA, Thielen and Shekhar (2002) found conditioned place aversion to the stimulation-paired context, after only five 
pairings of each type. Thus, stimulation of the BLA appears to be aversive, regardless of how it is applied. Because I did not test for conditioned place aversion until 45 pairings of each type had occurred, it is not known whether the rats would have shown a conditioned place aversion after just five stimulations. It is therefore possible that an aversion to the stimulation itself caused the conditioned place aversion. The conditioned anticipatory defensive responses, however, did not become evident until many more than five stimulations had occurred. The unconditioned stimulus for the conditioning of those responses was likely not the stimulations themselves, but rather some aspect of the resultant seizures. Further, as the anticipatory responses demonstrate that the seizures have an aversive component that served as an unconditioned stimulus, it is unlikely that the stimulation served uniquely as the unconditioned stimulus for the conditioned place aversion. At a minimum, the seizures contributed to that role.

The forceful convulsions produced by generalized amygdaloid-kindled seizures did not likely serve as an aversive US. Barnes et al. (2003) failed to demonstrate conditioned place aversion or preference for a context paired with anterior-neocortexkindled convulsions, nor did they find conditioned anticipatory defensive responses. Such convulsions are also forceful, but topologically different. A forceful convulsion, per se, does not therefore seem to be sufficiently aversive to produce the conditioned effects I observed.

Amygdaloid-kindled seizures could therefore be the relevant US. However, conditioned anticipatory responses did not appear until rats were having generalized seizures. If amygdaloid-kindled seizures, per se, were serving as the aversive US, those 
effects should have appeared much earlier in the experiment. A plausible hypothesis is thus that generalized amygdaloid-kindled seizures are the relevant aversive US.

However, generalized seizures produced by kindling of other limbic sites may be equivalent for that purpose. Barnes et al. (2005) kindled perirhinal cortex, which kindles at a rate similar to the amygdala, and found conditioned anticipatory defensive responses, and a conditioned place aversion. Ventral hippocampal kindling, which progresses much more slowly, failed to produce conditioned anticipatory defensive responses until rats had experienced many more seizures than were necessary with perirhinal kindling. Dorsal hippocampal kindling, which progresses even more slowly, failed to produce those conditioned effects.

It is also possible that generalized seizures from non-limbic sites could serve as an aversive US. Though Barnes et al. (2003) failed to find conditioned defensiveness or place aversion when they kindled the anterior neocortex, they did not continue kindling for a sufficient time for all of the rats to develop the second phase of neocortical kindling, in which generalized seizures occur (Burnham, 1978; Seidel \& Corcoran, 1986). Thus, it remains to be shown whether generalized seizures produced by anterior neocortical kindling can produce conditioned defensiveness in the present experimental paradigm.

If amygdaloid stimulations served as the US that produced conditioned place aversion, and generalized seizures served as the US that produced conditioned anticipatory responses, one would expect to find anticipatory responses, and not place aversion, if many generalized anterior neocortically kindled seizures were evoked. After receiving many stimulations to the anterior neocortex, rats did not show a conditioned place aversion (Barnes et al., 2003). Anterior neocortical stimulation therefore does not 
seem to be aversive in the same way that amygdaloid stimulation can be (Thielen \& Shekhar, 2002). However, it seems unlikely that each conditioned effect is produced by a different US, because the conditioned anticipatory responses and the conditioned place aversion are all defensive responses conditioned to the same environment.

Generalized seizures are likely the relevant aversive US for the conditioning of anticipatory defensive responses. It is possible that amygdaloid stimulation itself is a sufficiently aversive US to produce conditioned place aversion. At a minimum, however, it seems likely that the generalized amygdaloid-kindled seizures that the rats experienced also contributed to that role.

I suggest that the results contradict the interpretation that a generalized limbickindled seizure results in a loss of consciousness. One possibility is that such a seizure has a component wherein consciousness is intact that is sufficient to permit learning to occur. Another possibility is that such a seizure alters consciousness to a sufficiently mild degree that learning can occur. A third possibility is that a loss or alteration of consciousness, in and of itself, is sufficient as an aversive US.

The first possibility is unlikely. A period of intact consciousness would likely come very early in the seizure, when the rat is experiencing less than a stage 5 convulsion. If that early part of the seizure were a sufficient US, conditioned effects should have been evident much earlier than they were, and perhaps been reduced as the latency to a generalized convulsion decreased.

Most patients with temporal lobe epilepsy are at least partially aware of when they have had a seizure (Inoue \& Mihara, 1998; Kerling et al., 2006). Inoue and Mihara (1998) found that none of the 88 patients with temporal lobe epilepsy they tested recalled 
stimuli presented during partial seizures. However, the rats in my experiments did not experience a seizure until they had been in the CS+ for 30 sec; that contingency alone could account for conditioned effects, provided that the animals experienced some aversive aspect of having a seizure. They would thus not need to attend to the environment during a seizure, but would simply need to learn that the environment was predictive of a seizure.

It remains possible that a loss of consciousness is the aversive US. However, measurement of a rat's state of consciousness is not at all direct. Among kindling researchers, falling, or a loss of equilibrium, during a seizure is often taken as a sign of an impairment or loss of consciousness. It is rather difficult to test whether the concept is valid. I cannot therefore conclude whether a loss or impairment of consciousness per se served as the relevant aversive US.

I did not find any differences between rats with electrode tips in the BLA and those with electrode tips in the Central Nucleus of the amygdala. This could be because these two highly interconnected nuclei (Sah et al., 2003) cannot be functionally discriminated in the present paradigm. Indeed, both nuclei are involved in defensive behaviours, even if those behaviours are different (Killcross et al., 1997). Another possibility is that the stimulation activated approximately the same tissue, regardless of subtle differences in electrode placements. Though some electrode tips were found in the BLA and some in the Central Nucleus of the amygdala, the electrode tips were generally quite closely distributed. That is, BLA placements tended to be in the medial extent of the BLA, and Central Nucleus placements tended to be in the lateral extent of that nucleus. Given the size of current density distribution likely produced by the intensity of high 
stimulation (e.g., C. C. McIntyre \& Grill, 2001), stimulation via the electrode placements in the two groups may have activated amygdaloid nuclei similarly. Thus, the results should not necessarily be taken to indicate functional similarity between the BLA and Central Nucleus of the amygdala.

Rats kindled in the White context showed a greater contextual difference in defensiveness than did rats kindled in the Black context. It seems that the White context itself was anxiogenic. Indeed, bright light is understood to be anxiogenic for rats. It can enhance fear-potentiated startle, and that effect has been shown to be dependent on the BLA (Walker \& Davis, 1997). An anxiogenic effect of the White context could therefore have partially mitigated contextually conditioned defensiveness in the Black group, and enhanced it in the White group.

In contrast to the results of Freeman and Mikulka (1986), I did not find a difference in kindling rate between rats kindled in the White context and those kindled in the Black context. However, their contexts differed more than mine with respect to brightness, and I might have found such a contextual difference in kindling rate if my contexts were more similar to theirs. In any case, my failure to replicate their effect suggests that it is not easily produced.

Perhaps the most significant observation in my experiments is my failure to find any conditioned effects on ictal measures, in contrast to others (e.g., Barnes et al., 2001). One explanation is that subtle procedural differences mitigated the conditioning of the effects. Though my procedure was designed to closely replicate that of Barnes et al. (2001), there are some differences worth exploring. 
My parameters of stimulation were different. Whereas Barnes et al. (2001) used 1-sec trains of $60 \mathrm{~Hz}$ sine wave stimulation at $400 \mu \mathrm{A}$ RMS, I used a 1 sec train of 60 pp/s balanced biphasic square wave pulses, at $400 \mu \mathrm{A}$ base-to-peak. This difference should not be important, in my opinion. Both stimulation designs elicit $\mathrm{AD}$, and it is well established that kindling can occur using a variety of stimulation types as long as AD is elicited. As well, both stimulation designs used intensities substantially above typical ADTs for the amygdala.

On CS- trials, Barnes et al. (2001) triggered the switch on the stimulator, whereas I did not do so. Because they did not record EEG, Barnes et al. were able to disconnect the lead from their equipment for CS- trials, thus permitting them to trigger the stimulation switch. In contrast, I recorded EEG on all trials, necessitating an intact connection between the lead and the equipment. As triggering my, and likely their, stimulation switch makes a slightly audible sound, it is possible that the sound served as a conditioned stimulus predicting a seizure. In the switch tests, that sound was produced as seizures were elicited in each context. If the sound, as opposed to the context, served as the seizure-predicting CS, each rat's contextual conditioning history would have been irrelevant. However, my rats did apparently learn the predictive nature of the two contexts, because they developed contextually conditioned defensiveness. The slight clicking sound produced by triggering the switch may have served as an adjunct CS, but likely did not serve as the main seizure-predictive CS.

Whereas I kept the stimulation/recording lead attached to each rat throughout all trials, Barnes et al. (2001) removed the lead immediately after the (sham) stimulation was delivered. This occurred on both CS+ and CS- trials during conditioning. A contextually 
differential effect of removing the lead during the switch test would therefore be contingent upon differential sensitivity to some seizure-modulating effect of removing the lead. That is, removing the lead would have to have an anti-seizure effect to which the rats gained contextually conditioned tolerance. In humans, startling auditory stimuli or shaking by an experimenter near the onset of a seizure may have an anti-seizure effect (Zlutnick et al., 1975). It therefore seems possible that removing the lead could have such an effect on rats during kindling. If that effect is produced because of the surprising or disruptive nature of lead removal, rats may have become habituated to it in the CS+ after experiencing it many times. As such an anti-seizure effect would not have occurred in the CS- prior to the switch test, contextually conditioned habituation may have occurred, and the anti-seizure effect of removing the lead after stimulation would remain intact in the CS-.

The place-conditioning apparatus I used was slightly different from that of Barnes et al. (2001). It is unlikely that the lack of a central chamber would be relevant for anything other than the precision of the place preference test. Of note, though, is that my apparatus had bedding only in the White context. This likely increased the distinctiveness of the contexts, perhaps adding olfactory cues. It should therefore have strengthened any contextually conditioned effects. Ultimately, the rats in my experiments developed contextually conditioned defensiveness; they were able to associate the contexts with the events that occurred therein.

Contrary to the conclusions of Barnes et al. (2001), I suggest that conditioning is not fundamental to kindling. At a minimum, the conditioned effects on ictal measures found by Barnes et al. seem to be fragile. If my failure to replicate those effects can be 
explained by procedural differences, the effects are not sufficiently robust to be present in all kindling experiments or to have power as a potential mechanism of kindling.

In measuring $\mathrm{AD}$ and $\mathrm{ADT}$, I explicitly tested the hypothesis that the conditioning of convulsions found by Barnes et al. (e.g., 2001) was due to conditioned effects on AD and/or ADT. I did not find any conditioned effects on AD or ADT. Thus, I did not find support for that hypothesis. However, given that no conditioned effects on convulsions were found in the present experiment, my rejection of that hypothesis is limited. Ideally one would, while measuring EEG, replicate conditioned effects on convulsions, and test for conditioned effects on $\mathrm{AD}$ and $\mathrm{ADT}$. It remains ultimately unknown whether the latter would be found if the former were found.

There have been attempts to modify seizures in humans using conditioning techniques, which have been met with limited success and acceptance by the neurological community. The present failure to condition ictal responses certainly does not support such a use of conditioning techniques. Rather, interictal features of partial complex epilepsy with temporal lobe foci may be produced and modified by conditioning. That is, interictal emotionality, which may involve unusual affective value being assigned to various stimuli (Bear \& Fedio, 1977), may be produced by learned associations. Such emotionality could then be modified by conditioning techniques such as extinction.

In summary, using amygdaloid kindling, or some aspect thereof, as an unconditioned stimulus, I found evidence of conditioned interictal defensiveness, but not conditioned effects on ictal measures. The generalized seizures elicited by kindling of the amygdala were likely the relevant US in producing conditioned interictal defensiveness. My failure to replicate the conditioned effects on ictal measures found by Barnes et al. 
(2001) indicates that the effects are not as robust as previously thought. I conclude that conditioning is not fundamental to kindling. 


\section{References}

Adamec, R., \& Shallow, T. (2000). Effects of baseline anxiety on response to kindling of the right medial amygdala. Physiol Behav, 70(1-2), 67-80.

Adamec, R. E., \& Morgan, H. D. (1994). The effect of kindling of different nuclei in the left and right amygdala on anxiety in the rat. Physiol Behav, 55(1), 1-12.

Andrews, D. J., \& Schonfeld, W. H. (1992). Predictive factors for controlling seizures using a behavioural approach. Seizure, 1(2), 111-116.

Barnes, S. J., Hua, J. M., Pinel, J. P., Takahashi, A., \& Wig, G. S. (2005). Conditioned effects of kindling three different sites in the hippocampal complex of the rat. Behav Neurosci, 119(6), 1572-1579.

Barnes, S. J., Magyar, O., Pinel, J. P., \& Takahashi, A. (2004). Anticipating the attack: Temporal conditioning during amygdala kindling in rats. Behav Neurosci, 118(1), 89-96.

Barnes, S. J., Pinel, J. P., Francis, L. H., \& Wig, G. S. (2001). Conditioning of ictal and interictal behaviors in rats by amygdala kindling: Context as the conditional stimulus. Behav Neurosci, 115(5), 1065-1072.

Barnes, S. J., Pinel, J. P., Wee, E., Archambault, J., \& Ailon, T. (2006). Effects of expectation on amygdala-kindled convulsions and interictal behaviour in rats: Confirmation of a pavlovian mechanism. Eur J Neurosci, 24(3), 829-840.

Barnes, S. J., Pinel, J. P., Wig, G. S., Stuettgen, M. C., \& Holzel, C. H. (2003). Stimulation site determines the conditioned effects of kindling in rats: Anterior neocortex versus amygdala. Eur J Neurosci, 17(8), 1671-1679. 
Bear, D. M., \& Fedio, P. (1977). Quantitative analysis of interictal behavior in temporal lobe epilepsy. Arch Neurol, 34(8), 454-467.

Blanchard, E. B., \& Young, L. D. (1974). Clinical applications of biofeedback training. A review of evidence. Arch Gen Psychiatry, 30(5), 573-589.

Cataldo, M. F., Russo, D. C., \& Freeman, J. M. (1979). A behavior analysis approach to high-rate myoclonic seizures. J Autism Dev Disord, 9(4), 413-427.

Corcoran, M. E., Lanius, R., \& Duren, A. (1992). Reinforcing and punishing consequences of kindling. Epilepsy Res, 13(3), 179-186.

Cott, A., Pavloski, R. P., \& Black, A. H. (1979). Reducing epileptic seizures through operant conditioning of central nervous system activity: Procedural variables. Science, 203(4375), 73-75.

Egner, T., \& Sterman, M. B. (2006). Neurofeedback treatment of epilepsy: From basic rationale to practical application. Expert Rev Neurother, 6(2), 247-257.

Forster, F. M. (1969). Conditional reflexes and sensory-evoked epilepsy: The nature of the therapeutic process. Cond Reflex, 4(2), 103-114.

Forster, F. M. (1972). The classification and conditioning treatment of the reflex epilepsies. Int J Neurol, 9(1), 73-86.

Forster, F. M., Booker, H. E., \& Gascon, G. (1967). Conditioning in musicogenic epilepsy. Trans Am Neurol Assoc, 92, 236-237.

Forster, F. M., \& Campos, G. B. (1964). Conditioning factors in stroboscopic-induced seizures. Epilepsia, 59, 156-165.

Forster, F. M., Ptacek, L. J., \& Peterson, W. G. (1965). Auditory clicks in extinction of stroboscope-induced seizures. Epilepsia, 6(3), 217-225. 
Forster, F. M., Ptacek, L. J., Peterson, W. G., Chun, R. W., Bengzon, A. R., \& Campos, G. B. (1964a). Stroboscopic-induced seizure discharges. Modification by extinction techniques. Arch Neurol, 11, 603-608.

Forster, F. M., Ptacek, L. J., Peterson, W. G., Chun, R. W., \& Campos, G. B. (1964b). Stroboscopic induced seizures altered by extinction techniques. Trans Am Neurol Assoc, 89, 136-139.

Freeman, F. G., \& Mikulka, P. J. (1986). Differential conditioning of environmental cues with amygdala kindling. Epilepsia, 27(3), 189-193.

Gilbert, T. H., Hannesson, D. K., \& Corcoran, M. E. (2000). Hippocampal kindled seizures impair spatial cognition in the morris water maze. Epilepsy Res, 38(2-3), 115-125.

Gilbert, T. H., McNamara, R. K., \& Corcoran, M. E. (1996). Kindling of hippocampal field ca1 impairs spatial learning and retention in the morris water maze. Behav Brain Res, 82(1), 57-66.

Goddard, G. V. (1964). Functions of the amygdala. Psychol Bull, 62, 89-109.

Goddard, G. V. (1967). Development of epileptic seizures through brain stimulation at low intensity. Nature, 214(92), 1020-1021.

Goddard, G. V., McIntyre, D. C., \& Leech, C. K. (1969). A permanent change in brain function resulting from daily electrical stimulation. Exp Neurol, 25(3), 295-330.

Goldstein, M. L. (1965). Effects of hippocampal, amygdala, hypothalamic and parietal lesions on a classically conditioned fear response. Psychol Rep, 16, 211-219.

Hannesson, D. K., \& Corcoran, M. E. (2000). The mnemonic effects of kindling. Neurosci Biobehav Rev, 24(7), 725-751. 
Hannesson, D. K., Howland, J., Pollock, M., Mohapel, P., Wallace, A. E., \& Corcoran, M. E. (2001). Dorsal hippocampal kindling produces a selective and enduring disruption of hippocampally mediated behavior. J Neurosci, 21(12), 4443-4450.

Hannesson, D. K., Wallace, A. E., Pollock, M., Corley, S., Mohapel, P., \& Corcoran, M.

E. (2004). The relation between extent of dorsal hippocampal kindling and delayed-match-to-place performance in the morris water maze. Epilepsy Res, 58(2-3), 145-154.

Helmstaedter, C., Sonntag-Dillender, M., Hoppe, C., \& Elger, C. E. (2004). Depressed mood and memory impairment in temporal lobe epilepsy as a function of focus lateralization and localization. Epilepsy Behav, 5(5), 696-701.

Huberty, C. J., \& Morris, J. D. (1989). Multivariate analysis versus multiple univariate analyses. PsycholBull, 105, 302-308.

Hunsperger, R. W. (1963). [affective behavior patterns elicited by electrical stimulation of the brain stem and forebrain.]. J Physiol (Paris), 55, 45-98.

Inoue, Y., \& Mihara, T. (1998). Awareness and responsiveness during partial seizures. Epilepsia, 39 Suppl 5, 7-10.

Kalynchuk, L. E. (2000). Long-term amygdala kindling in rats as a model for the study of interictal emotionality in temporal lobe epilepsy. Neurosci Biobehav Rev, 24(7), 691-704.

Kalynchuk, L. E., Pinel, J. P., \& Treit, D. (1998a). Long-term kindling and interictal emotionality in rats: Effect of stimulation site. Brain Res, 779(1-2), 149-157.

Kalynchuk, L. E., Pinel, J. P., \& Treit, D. (1999). Characterization of the defensive nature of kindling-induced emotionality. Behav Neurosci, 113(4), 766-775. 
Kalynchuk, L. E., Pinel, J. P., Treit, D., Barnes, S. J., McEachern, J. C., \& Kippin, T. E. (1998b). Persistence of the interictal emotionality produced by long-term amygdala kindling in rats. Neuroscience, 85(4), 1311-1319.

Kalynchuk, L. E., Pinel, J. P., Treit, D., \& Kippin, T. E. (1997). Changes in emotional behavior produced by long-term amygdala kindling in rats. Biol Psychiatry, 41(4), 438-451.

Lowenstein, D. H. (2005). Seizures and epilepsy. In D.L. Kasper, \& T.R. Harrison. Harrison's principles of internal medicine (16th ed.). New York: McGraw-Hill, Medical Pub. Division.

Kerling, F., Mueller, S., Pauli, E., \& Stefan, H. (2006). When do patients forget their seizures? An electroclinical study. Epilepsy Behav, 9(2), 281-285.

Killcross, S., Robbins, T. W., \& Everitt, B. J. (1997). Different types of fear-conditioned behaviour mediated by separate nuclei within amygdala. Nature, 388(6640), 377380.

Kluver, H., \& Bucy, P. (1939). Preliminary analysis of functions of the temporal lobes in monkeys. Archives of Neurology \& Psychiatry (Chicago) 42 1939, 979-1000.

Krafft, K. M., \& Poling, A. D. (1982). Behavioral treatments of epilepsy: Methodological characteristics and problems of published studies. Appl Res Ment Retard, 3(2), 151-162.

Leung, L. S., Brzozowski, D., \& Shen, B. (1996). Partial hippocampal kindling affects retention but not acquisition and place but not cue tasks on the radial arm maze. Behav Neurosci, 110(5), 1017-1024. 
Livingston, K. E., Hornykiewicz, O., \& Society for Neuroscience. (1978). Limbic mechanisms: The continuing evolution of the limbic system concept: [proceedings of the limbic system symposium held at the university of toronto, ontario, canada, november 5-6, 1976, as a satellite to the sixth annual meeting of the society of neuroscience]. New York: Plenum Press.

Lopes da Silva, F. H., Gorter, J. A., \& Wadman, W. J. (1986). Kindling of the hippocampus induces spatial memory deficits in the rat. Neurosci Lett, 63(2), 115120.

Maclean, P. D., \& Delgado, J. M. (1953). Electrical and chemical stimulation of frontotemporal portion of limbic system in the waking animal. Electroencephalogr Clin Neurophysiol Suppl, 5(1), 91-100.

McIntyre, C. C., \& Grill, W. M. (2001). Finite element analysis of the current-density and electric field generated by metal microelectrodes. Ann Biomed Eng, 29(3), 227235.

McIntyre, D. C. (1970). Differential amnestic effect of cortical vs. Amygdaloid elicited convulsions in rats. Physiol Behav, 5(7), 747-753.

McIntyre, D. C. (1979). Effects of focal vs generalized kindled convulsions from anterior neocortex or amygdala on cer acquisition in rats. Physiol Behav, 23(5), 855-859.

McIntyre, D. C., \& Molino, A. (1972). Amygdala lesions and cer learning: Long term effect of kindling. Physiol Behav, 8(6), 1055-1058.

Mohapel, P., Dufresne, C., Kelly, M. E., \& McIntyre, D. C. (1996). Differential sensitivity of various temporal lobe structures in the rat to kindling and status epilepticus induction. Epilepsy Res, 23(3), 179-187. 
Nadkarni, S., LaJoie, J., \& Devinsky, O. (2005). Current treatments of epilepsy. Neurology, 64(12 Suppl 3), S2-11.

Ng, B. Y. (2002). Psychiatric aspects of self-induced epileptic seizures. Aust N Z J Psychiatry, 36(4), 534-543.

Paredes, R. G., Muzzi, G., Aguirre, E., \& Romero, V. (2000). Can a generalized kindling seizure induce a reward state? Epilepsy Res, 38(2-3), 249-257.

Paxinos, G. (1995). The rat nervous system (2nd ed.). San Diego: Academic Press.

Paxinos, G., \& Watson, C. (1997). The Rat Brain in Stereotaxic Coordinates (Compact 3rd ed.). San Diego: Academic Press.

Figure 1 Reprinted from The Rat Brain in Stereotaxic Coordinates (Compact 3rd ed.), Paxinos, G. \& Watson, C., Figures 25-30, Copyright (1997), with permission from Elsevier.

Paxinos, G., \& Watson, C. (1998). The rat brain in stereotaxic coordinates (4th ed.). San Diego: Academic Press.

Pinel, J. P., \& Rovner, L. I. (1978). Experimental epileptogenesis: Kindling-induced epilepsy in rats. Exp Neurol, 58(2), 190-202.

Quy, R. J., Hutt, S. J., \& Forrest, S. (1979). Sensorimotor rhythm feedback training and epilepsy: Some methodological and conceptual issues. Biol Psychol, 9(2), 129149.

Racine, R. J. (1972a). Modification of seizure activity by electrical stimulation. I. Afterdischarge threshold. Electroencephalogr Clin Neurophysiol, 32(3), 269-279.

Racine, R. J. (1972b). Modification of seizure activity by electrical stimulation. Ii. Motor seizure. Electroencephalogr Clin Neurophysiol, 32(3), 281-294. 
Ritaccio, A. L., Singh, A., \& Devinsky, O. (2002). Cognition-induced epilepsy. Epilepsy Behav, 3(6), 496-501.

Robinson, E. (1963). Effect of amygdalectomy on fear-motivated behavior in rats. $J$ Comp Physiol Psychol, 56, 814-820.

Sah, P., Faber, E. S., Lopez De Armentia, M., \& Power, J. (2003). The amygdaloid complex: Anatomy and physiology. Physiol Rev, 83(3), 803-834.

Saucier, D. M., \& Corcoran, M. E. (2003). Kindling. In L. Nadel (Ed.) Encyclopedia of Cognitive Neuroscience. London: Nature Publishing Group. p660-666.

Seidel, W. T., \& Corcoran, M. E. (1986). Relations between amygdaloid and anterior neocortical kindling. Brain Res, 385(2), 375-378.

Skinner, B. F. (1934). The extinction of chained reflexes. Proc Natl Acad Sci U S A, 20(4), 234-237.

So, E. L. (2006). Value and limitations of seizure semiology in localizing seizure onset. $J$ Clin Neurophysiol, 23(4), 353-357.

Sterman, M. B. (2000). Basic concepts and clinical findings in the treatment of seizure disorders with eeg operant conditioning. Clin Electroencephalogr, 31(1), 45-55.

Sterman, M. B., \& Egner, T. (2006). Foundation and practice of neurofeedback for the treatment of epilepsy. Appl Psychophysiol Biofeedback, 31(1), 21-35.

Thielen, S. K., \& Shekhar, A. (2002). Amygdala priming results in conditioned place avoidance. Pharmacol Biochem Behav, 71(3), 401-406.

Walker, D. L., \& Davis, M. (1997). Double dissociation between the involvement of the bed nucleus of the stria terminalis and the central nucleus of the amygdala in 
startle increases produced by conditioned versus unconditioned fear. J Neurosci, 17(23), 9375-9383.

Wig, G. S., Barnes, S. J., \& Pinel, J. P. (2002). Conditioning of a flavor aversion in rats by amygdala kindling. Behav Neurosci, 116(2), 347-350.

Wintink, A. J., Young, N. A., Davis, A. C., Gregus, A., \& Kalynchuk, L. E. (2003). Kindling-induced emotional behavior in male and female rats. Behav Neurosci, 117(3), 632-640.

Wolf, P. (2002). The role of nonpharmaceutic conservative interventions in the treatment and secondary prevention of epilepsy. Epilepsia, 43 Suppl 9, 2-5.

World Health Organization (2001). Epilepsy: aetiogy, epidemiology and prognosis.

Retrieved November 23, 2006, from http://www.who.int/mediacentre/factsheets/fs165/en/

Wyler, A. R., Lockard, J. S., Ward, A. A., Jr., \& Finch, C. A. (1976). Conditioned eeg desynchronization and seizure occurrence in patients. Electroencephalogr Clin Neurophysiol, 41(5), 501-512.

Zlutnick, S., Mayville, W. J., \& Moffat, S. (1975). Modification of seizure disorders: The interruption of behavioral chains. J Appl Behav Anal, 8(1), 1-12. 


\section{Appendix 1}

\section{Permission to Reprint Copyrighted Material}

This electronic mail was received February 2, 2007, from Stephanie Smith

(ST.smith@elsevier.com), a representative of Elsevier Ltd.

We hereby grant you permission to reprint the aforementioned material

at

no charge in your thesis subject to the following conditions:

1. If any part of the material to be used (for example, figures) has appeared in our publication with credit or acknowledgement to another source, permission must also be sought from that source. If such permission is not obtained then that material may not be included in your publication/copies.

2. Suitable acknowledgment to the source must be made, either as a footnote or in a reference list at the end of your publication, as follows:

"Reprinted from Publication title, Vol number, Author(s), Title of article, Pages No., Copyright (Year), with permission from Elsevier".

3. Your thesis may be submitted to your institution in either print or electronic form.

4. Reproduction of this material is confined to the purpose for which permission is hereby given.

5. This permission is granted for non-exclusive world English rights only. For other languages please reapply separately for each one required. Permission excludes use in an electronic form. Should you have a specific electronic project in mind please reapply for permission.

6. This includes permission for the Library and Archives of Canada to supply single copies, on demand, of the complete thesis. Should your

thesis be published commercially, please reapply for permission.

Yours sincerely

Steph Smith

Rights Assistant

Elsevier Ltd

The Boulevard

Langford Lane

Kidlington

oxford 0X5 1GB 


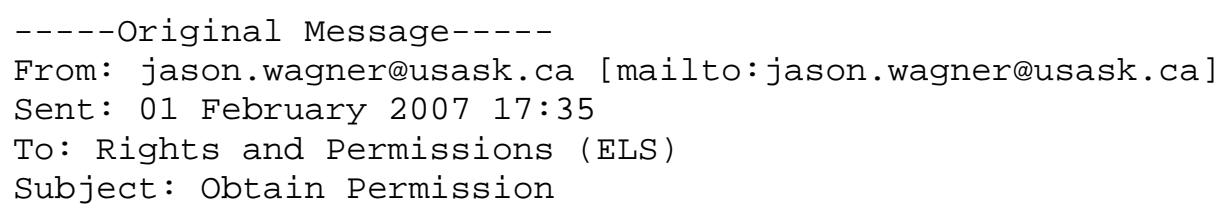

This Email was sent from the Elsevier Corporate Web Site and is related to Obtain Permission form:

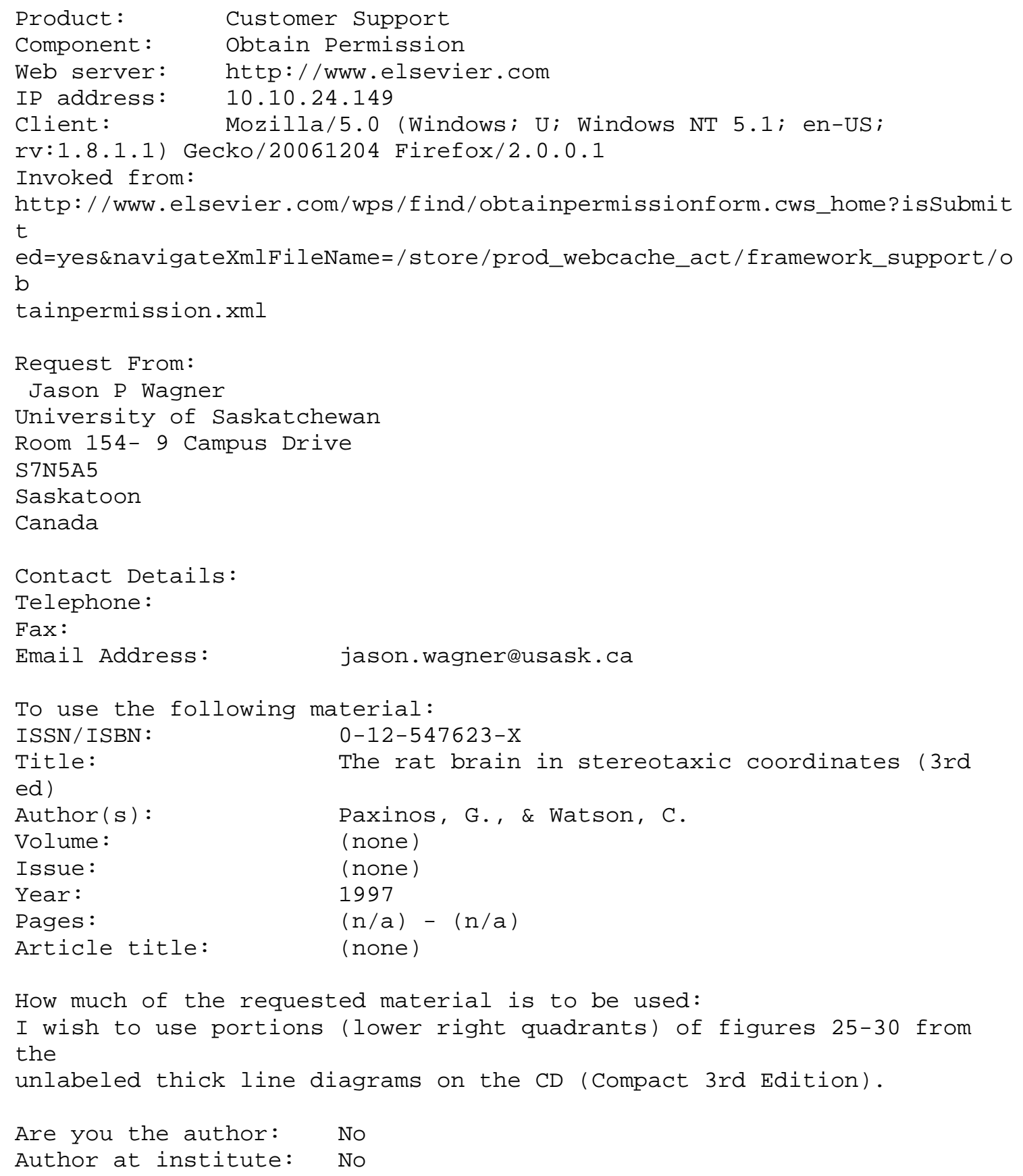


How/where will the requested material be used:

[how_used]

Details:

I wish to use this material in a single figure in my M.A. (Psychology) thesis, to show electrode tip placements.

Additional Info:

- end -

For further info regarding this automatic email, please contact:

WEB APPLICATIONS TEAM ( esweb.admin@elsevier.co.uk ) 